\title{
Two new Liolaemus lizards from the Andean highlands of Southern Chile (Squamata, Iguania, Liolaemidae)
}

\author{
Jaime Troncoso-Palacios', Hugo A. Diaz ${ }^{2,3}$, German I. Puas ${ }^{4,5}$, \\ Edvin Riveros-Riffo ${ }^{6}$, Alvaro A. Elorza ${ }^{4,5}$
}

I Programa de Fisiologia y Biofisica, Instituto de Ciencias Biomedicas (ICBM), Facultad de Medicina, Universidad de Chile, Independencia 1027, Santiago, Chile 2 Departamento de Ciencias Ecologicas, Facultad de Ciencias, Universidad de Chile, Las Palmeras 3425, Santiago, Chile 3 Departamento de Ciencias Biologicas Animales, Facultad de Ciencias Veterinarias y Pecuarias, Universidad de Chile, Santa Rosa 11735, Santiago, Chile 4 Centro de Investigaciones Biomedicas, Facultad de Ciencias Biologicas y Facultad de Medicina, Universidad Andres Bello, Republica 239, Santiago, Chile 5 Instituto Milenio de Inmunologia e Inmunoterapia, Portugal 49, Santiago, Chile 6 Red de Observadores de Aves y Vida Silvestre de Chile, Julio Prado 1144 Dpto. 31, Providencia, Chile

Corresponding author: Jaime Troncoso-Palacios (jtroncosopalacios@gmail.com)

Academic editor: A. Bauer | Received 12 June 2016 | Accepted 16 October 2016 | Published 16 November 2016 http://zoobank.org/0910B0A2-3CA9-4C37-B6AD-1DEB92ADDFFD

Citation: Troncoso-Palacios J, Diaz HA, Puas GI, Riveros-Riffo E, Elorza AA (2016) Two new Liolaemus lizards from the Andean highlands of Southern Chile (Squamata, Iguania, Liolaemidae). ZooKeys 632: 121-146. doi: 10.3897/ zookeys.632.9528

\begin{abstract}
Liolaemus is a diverse genus of lizards, subdivided into two subgenera: Liolaemus (sensu stricto) and Eulaemus, distributed mainly in Chile and Argentina. The L. elongatus-kriegi complex is the most diverse group within Liolaemus (sensu stricto), especially the species closely related to L. elongatus, which form a clade currently comprising nine species. Several Chilean species of this group have been recently described, mainly from volcanoes and poorly explored mountains. Here molecular and morphological evidence are provided for a new species of the L. elongatus clade, which is characterized by its small size and lack of dorsal pattern, unusual features for the species of this group of lizards. Additionally, the lack of precloacal pores in males of Liolaemus (sensu stricto) is a trait found in few species, which do not constitute a monophyletic group. A second new southern Chilean species is also described, without precloacal pores and supported by molecular phylogenetics to be related to Liolaemus villaricensis. Both new species were found in the same locality, near a lake located in a pre-Andean zone with Araucaria and Nothofagus forest.
\end{abstract}

Copyright Jaime Troncoso-Palacios et al. This is an open access article distributed under the terms of the Creative Commons Attribution License (CC BY 4.0), which permits unrestricted use, distribution, and reproduction in any medium, provided the original author and source are credited. 
The two species are dedicated to prominent Lonkos (tribal chiefs) of the Mapuche and Pehuenche people: Janequeo and Leftraru. Additionally, the phylogenetic results suggest that L. lonquimayensis is a synonym of L. elongatus.

\section{Keywords}

Cytochrome b, Liolaemus elongatus, L. villaricensis, mtDNA, new species, precloacal pores

\section{Introduction}

Liolaemus is one of the most diverse genera of lizards, including 252 species (Uetz and Hošek 2015) that are grouped into two subgenera: Liolaemus (sensu stricto) and Eulaemus (e.g. Laurent 1985, Schulte et al. 2000), distributed mainly in Chile and Argentina (Abdala and Quinteros 2014). Recently, the exploration of volcanoes and rarely visited highlands in central and southern Chile has led to the discovery and description of several new species of lizards (Escobar-Huerta et al. 2015, Esquerré et al. 2013, 2014, TroncosoPalacios et al. 2015, 2016), most of them belonging to the L. elongatus-kriegi complex of the Liolaemus subgenus. This complex consists of four clades: the punmahuida, petrophilus, kriegi and elongatus groups (Avila et al. 2004, 2012, Morando et al. 2003). The $L$. elongatus clade was characterized by Avila et al. (2015) as a group of lizards of medium to large size, having long-tails, with reduced sexual dichromatism, viviparous, insectivorous, and almost exclusively saxicolous. Currently, this clade comprises nine species distributed in central and southern Chile and Argentina: L. antumalguen Avila, Morando, Perez and Sites, 2010; L. burmeisteri Avila, Pérez, Medina, Sites and Morando, 2012; L. carlosgarini Esquerré, Núñez and Scolaro, 2013; L. choique; L. elongatus Koslowsky, 1896; L. shitan; L. smang Abdala, Quinteros, Scrocchi and Stazzonelli, 2010; L. crandalli Avila, Medina, Fulvio-Pérez, Sites and Morando, 2015; and L. lonquimayensis Escobar-Huerta, Santibáńez-Toro and Ortiz, 2015. Although several new species have been described in recent years, it has been suggested that the diversity within the L. elongatus-kriegi complex is underestimated and the number of species could be doubled (Morando et al. 2003).

The males of most Liolaemus species have precloacal pores (Esquerré et al. 2013) and these are extensively used as taxonomic characters and for sex determination (Lobo 2005; Valdecantos et al. 2014). In fact, Esquerré et al. (2013) listed only five species of Liolaemus (sensu stricto) which lack precloacal pores, to which can now be added two newly described species: L. chavin Aguilar, Wood, Cusi, Guzmán, Huari, Lundberg, Mortensen, Ramírez, Robles, Suárez, Ticona, Vargas, Venegas \& Sites, 2013, and L. tregenzai Pincheira-Donoso \& Scolaro, 2007.

In a field campaign to southern Chile in January 2014, two sympatric species of Liolaemus were found which cannot be assigned to any known species. Here molecular and morphological evidence for a new species of the L. elongatus clade are provided. Molecular and morphological evidence are also given for another new species of Liolaemus (sensu stricto), which is the first species closely related to L. villaricensis Müller \& Hellmich, 1932, based on molecular phylogeny. 


\section{Materials and methods}

Morphological data and analyses. Morphological characters were examined according to Etheridge (1995), Lobo (2005) and Avila et al. (2010, 2012). Body measurements were made with a digital vernier caliper $(0.02 \mathrm{~mm}$ precision $)$. Measurements are provided as mean \pm standard deviation $(\mathrm{x} \pm \mathrm{SD})$. Scales were observed with different magnifying lenses. The scale characterization and measurements were recorded on the right side of the specimen, unless otherwise indicated. Dorsal scales were counted between the occiput and the level of the anterior border of the hind limbs. Ventral scales were counted from mental scale to the anterior margin of the cloacal opening. Stomach and intestinal contents were analyzed under a binocular stereoscope for one specimen of each new species. Specimens were collected in four field campaigns: January 2014, February 2015, January 2016 and September 2016. Both species are characterized by their low abundance and, to our knowledge, by highly restricted distributions. Despite four field campaigns, we only were able to collect seven specimens for each new species. All specimens were sexed through internal examination of testes or oocytes/embryos. We examined specimens of all Chilean species currently considered within the Liolaemus elongatus clade, including nine adult specimens of $L$. carlosgarini, six adult specimens of $L$. cf. elongatus and eleven adult specimens of $L$. scorialis; plus six adult specimens of $L$. villaricensis Müller and Hellmich, 1932. Additional data for eight adult specimens of L. carlosgarini were taken from Esquerré et al. (2013) and additional data for two adult specimens of $L$. villaricensis were taken from literature (Hellmich 1934). The specimens examined are listed in Suppl. material 1: Appendix I. Acronyms used in this work are: MZUC (Colección del Museo de Zoología de la Universidad de Concepción), MRC (Museo de Historia Natural de Concepción) and SSUC (Colección Patricio Sánchez Reyes, Pontificia Universidad Católica de Chile). Data for all species endemic to Argentina were taken from literature as follow. Liolaemus choique; L. shitan; and L. smaug was taken from Abdala et al. (2010). Data for L. antumalguen were taken from Avila et al. (2010), data for L. burmeisteri was taken from Avila et al. (2012) and data for L. crandal$l i$ were taken from Avila et al. (2015). Data for $L$. coeruleus Cei and Ortiz, 1983; and $L$. neuquensis Müller and Hellmich, 1939; were taken from Scolaro et al. (2007). Data for L. punmahuida Avila, Pérez and Morando, 2003; were taken from Avila et al. (2003). Data for L. tregenzai were taken from Pincheira-Donoso and Scolaro (2007). For the diagnosis, we performed a statistical analysis with data taken from all adult specimens directly examined (Suppl. material 1: Appendix I) plus data taken from published data set of $L$. antumalguen, L. carlosgarini and L. lonquimayensis. For the statistical analysis, we applied a Kolmogorov-Smirnov test to verify data normality, a subsequent t-test or Mann- Whitney U test was used if data passed or failed the normality test, respectively, to compare each variable. The statistical results are provided only when the differences were significant. Additionally, we performed a principal component analysis (PCA) to visualize and discriminate species in the morphological space, using the following variables: head length, head width, head height, snout-vent length (SVL), axilla-groin distance (AGD), arm length, foot length, midbody scales (SAMB), dorsal scales, ventral 
scales, supralabial scales, infralabial scales and fourth toe lamellae. This was performed with FactoMineR and R6 packages in RStudio and missing data were previously imputed with missMDA (RStudio Team 2015). Eigenvalues and the correlation of each variable with each of the first three PCs are provided in Appendices III and IV. For species that we did not examine and for which no published data sets exist, we performed a diagnosis based in the scale count ranges and SVL range, following the diagnoses previously published for the descriptions of Liolaemus included in this work (Abdala et al. 2010, Avila et al. 2010, 2012, 2015, Escobar-Huerta et al. 2015, Esquerré et al. 2013). Color pattern features were used as qualitative features of diagnosis between the two new species and all related species.

Genomic DNA purification, PCR amplification, and Sequencing. Samples from liver and thigh muscle were obtained from ethanol-fixed lizards and subjected to a rehydration process according to Coura (2005). Samples were washed twice in distilled water for $5 \mathrm{~min}$ at $55^{\circ} \mathrm{C}$ to remove the fixative and then rehydrated with 1x Tris/EDTA for $5 \mathrm{~min}$ at $55^{\circ} \mathrm{C}$ and then with $1 \mathrm{M}$ Tris $\mathrm{pH} 7.5$, at $55^{\circ} \mathrm{C}$ overnight, followed immediately by digestion with proteinase $\mathrm{K}(20 \mathrm{mg} / \mathrm{ml})$ at $55^{\circ} \mathrm{C}$ overnight. Genomic DNA isolation (mitochondrial and nuclear) was done with the Wizard $^{\oplus}$ Genomic DNA Purification kit (Cat \# A1120, Promega, USA) following manufacturer's instructions. The mitochondrial gene $C y t-b$ was amplified from total DNA through two phase conventional PCR with the primers GLUDGL (5'-TGA CTT GAA RAA CCA YCG TTG-3') and CB3 (5'-GGC AAA TAG GAA RTA TCA TTC-3'), reported in Torres- Pérez et al. (2009), to generate a 665 bp amplicon. PCR reactions were performed with the SapphireAmp ${ }^{\oplus}$ Fast PCR Master Mix (Cat \# RR350A, Takara Clontech, USA) using 100 ng of total genomic DNA as a template and following the instruction manual. Two-phase PCR cycling was as follow: Phase 1, initial $98^{\circ} \mathrm{C}$ denaturation for 3 min, then 5 cycles of $98^{\circ} \mathrm{C}$ denaturation for $30 \mathrm{~s}, 47^{\circ} \mathrm{C}$ annealing for $45 \mathrm{~s}$ and $72{ }^{\circ} \mathrm{C}$ extension for $45 \mathrm{~s}$. The Phase 2, next 40 cycles of $98{ }^{\circ} \mathrm{C}$ denaturation for $30 \mathrm{~s}, 58^{\circ} \mathrm{C}$ annealing for $45 \mathrm{~s}$ and $72{ }^{\circ} \mathrm{C}$ extension for $45 \mathrm{~s}$. A final $72{ }^{\circ} \mathrm{C}$ extension step for $5 \mathrm{~min}$ was added to finish the PCR. The 665 bp PCR amplicon was checked by DNA electrophoresis on a $1 \%$ agarose gel in $1 \mathrm{x}$ Tris-Acetate-EDTA (TAE) buffer. The amplicons were purified with the E.Z.N.A. ${ }^{\circledR}$ Cycle-Pure Kit (Cat \# D6492-02, Omega Biotek, USA) and sent for capillary sequencing to Macrogen, Korea.

Phylogenetic reconstruction. The GenBank accession numbers of the Cyt-b mitochondrial loci sequences generated in this study and the sequences obtained from GenBank are indicated in Suppl. material 1: Appendix II. Additionally, Gustavo Escobar-Huerta sent us the Cyt-b sequences of the type series of Liolaemus lonquimayensis (MZUC 40365-68). Cesar Aguilar and Jack Walter Sites Jr. sent us the Cyt-b sequence of one of the two specimens of Liolaemus sp.2 included in the phylogeny (SSUC Re 716). One hundred sixteen nucleotide sequences used in the analysis were aligned using MUSCLE (Edgar 2004). JModelTest v2.1.7 (Darriba et al. 2012, Guidon and Gascuel 2003) was used to select an appropriate substitution model $(H K Y+G+I)$, based on both the BIC and AIC indices. Bayesian inference (BI) analyses were performed with 
MrBayes v3.1.5 (Ronquist and Huelsenbeck 2003). Two independent analyses, each consisting of two groups of four chains that ran independently, were run for $10.0 \times 10^{6}$ generations and at sample frequency of 1000 using default priors. Phymaturus vociferator Pincheira-Donoso, 2004, was selected as the outgroup. Twenty-five percent of samples were discarded as burn-in when calculating the convergence diagnostic, assessed by examining values of average standard deviation of the Potential Scale Reduction Factor (PSRF $=1.000$ for all parameters) (Gelmar and Rubin 1992) and the minimum and average Estimated Sample Size (ESS $>4000$ for all parameters). The nodes were considered as strongly supported when pp $\geq 0.95$ (Huelsenbeck and Ronquist 2001). Additionally, a maximum likelihood phylogenetic analysis (ML) was performed with 1000 bootstrap replicates and calculated the average uncorrected pairwise difference (p-distance) using MEGA v6.06 (Tamura et al. 2013). Nodes with a bootstrap value $\geq$ 95\% were considered as strongly supported (Felsenstein and Kishino 1993).

\section{Results}

In our BI phylogeny (Fig. 1), the first species described in this work is found to be a member of the Liolaemus elongatus clade, which is strongly supported ( $\mathrm{pp}=0.99)$ and includes $L$. antumalguen, L. burmeisteri, L. choique, L. elongatus, L. lonquimayensis, $L$. shitan, L. smang, the species described here, an unidentified Liolaemus from Chillán and two candidate species (L. sp. 6 and $L$.sp. 7 ) previously suggested by Morando et al. (2003). In the ML phylogeny (Fig. 2) the L. elongatus clade was recovered with moderate support (bootstrap $=88 \%$ ) but with the same composition. In both analyses the first species described here is found as sister taxon of the clade L. elongatus + L. lonquimayensis $+L$. shitan ( $\mathrm{pp}=0.99$ and bootstrap $=74 \%$, respectively). The topology found in the ML phylogeny (Fig. 2) is very similar to the topology found in the BI phylogeny, but curiously $L$. petrophilus was found outside of the remainder of the L. petrophilus clade, which has low support (bootstrap $=48 \%$ ). In both, ML and BI phylogenies, $L$. shitan and $L$. lonquimayensis appear to be conspecific with L. elongatus; and also $L$. sp. 7 and $L$. antumalguen appear to be conspecifics. The addition of other species of the elongatus clade to the phylogeny might resolve these issues (see Discussion). Average uncorrected pairwise distance between the first new species and the clade $L$. elongatus + L. lonquimayensis + L. shitan is $3.4 \%$, consistent with a $3 \%$ divergence previously proposed for identification of candidate species in Liolaemus (Breitman et al. 2012).

The second species described here is found to be the sister species of Liolaemus villaricensis in both analysis (BI pp $=1.00$, ML bootstrap $=99 \%$, Figs 1 and 2), being the first species identified as closely related to this taxon based on molecular phylogeny. Average uncorrected pairwise distance between the species is $7.3 \%$, more than double that value proposed for identification of candidate species (Breitman et al. 2012).

In regards to the PCA analysis, only the first three principal components (PCs) account each more than $10 \%$ of the variation (Suppl. material 1: Appendix III). PC1 is mainly positively correlated with variation in morphological measures (SVL, head 


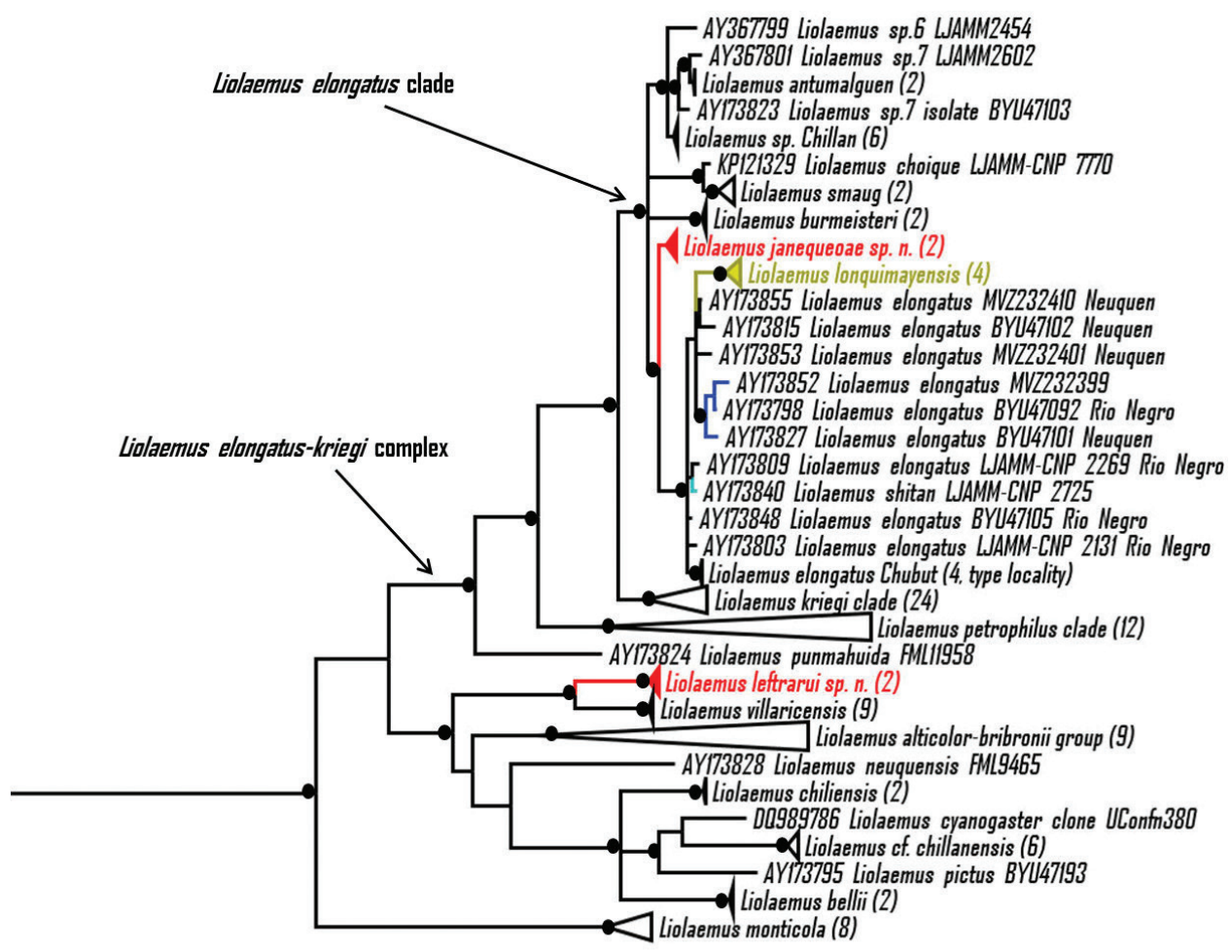

0.04

Figure I. Bayesian inference of phylogeny (BI) tree based on $C y t-b$, showing phylogenetic relationships of Liolaemus janequeoae sp. n. and L. leftrarui sp. n. (in red) (HKY+G+I). Liolaemus shitan is in light green, L. lonquimayensis is in yellow and L. elongatus samples used by Escobar-Huerta et al. (2015) are in blue. Posterior probability $\geq 0.95$ are indicated with a black dot. Numbers in parentheses indicate the amount of collapsed sequences. Scale shows the number of changes per site.

length, head width, axilla-groin distance and foot length, Suppl. material 1: Appendix IV, Fig. 3). PC2 is mainly positively correlated with the number of supralabials, fourth toe lamellae, infralabials and negatively correlated to the dorsal scales (Appendix IV, Fig. 3). PC3 is positively correlated mainly with the ventral, midbody and dorsal scale counts. The first species described here does not overlap in morphological space with $L$. elongatus (Fig. 3), found as its most closely related species in both BI and ML phylogenies (Figs 1 and 2). The second species described here marginally overlaps with L. villaricensis in morphological space when ellipses (95\% confidence interval around the centroid for each species) are generated with the first two PCs (Fig. 3). However, the second new species overlaps almost completely in morphological space with $L$. villaricensis, $L$. cf. chillanensis and L. scorialis when ellipses are generated with the second and third PCs (Fig. 3). Nevertheless, the second species described here is not closely related to $L$. cf. chillanensis (Figs 1 and 2) or L. scorialis, a member of the L. elongatus-kriegi clade (D. Esquerré, 


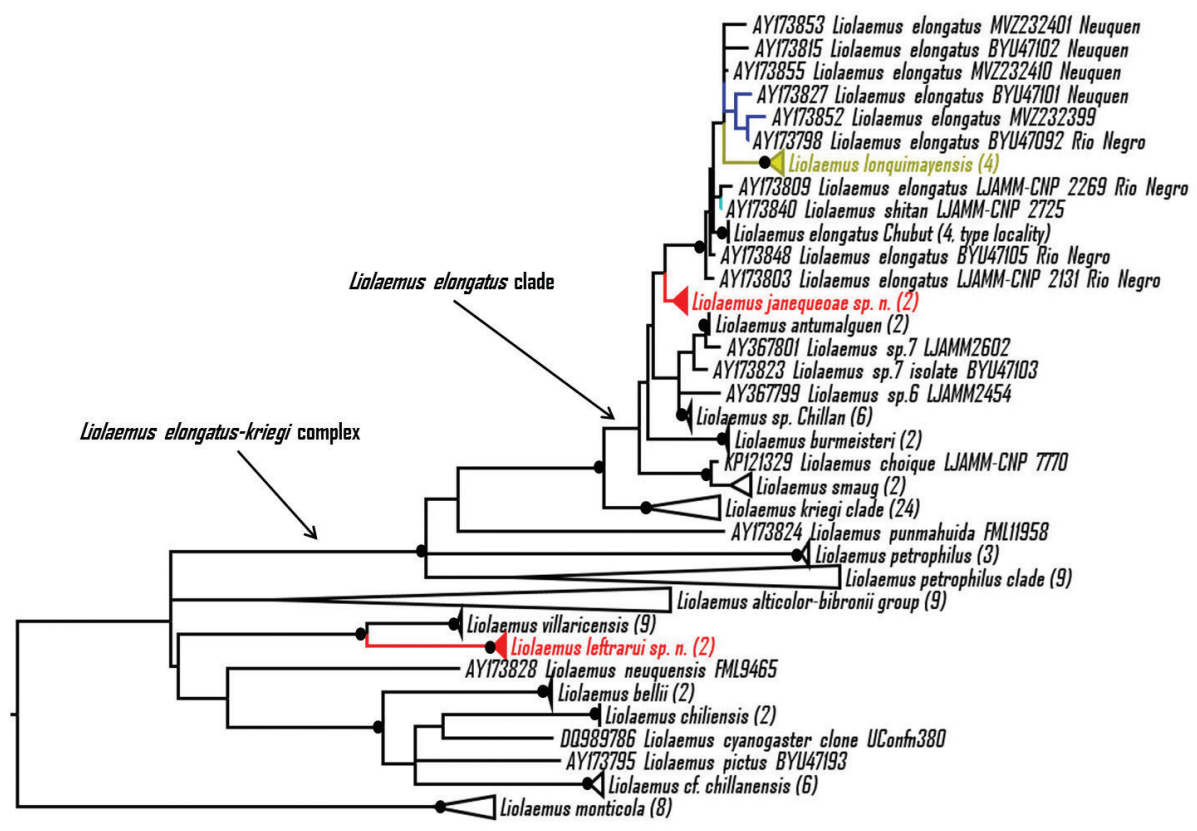

0.04

Figure 2. Maximum likelihood phylogeny (ML) tree based on $C y t-b$, showing phylogenetic relationships of Liolaemus janequeoae sp. n. and L. leftrarui sp. n. (in red) (HKY+G+I). Liolaemus shitan is in light green, L. lonquimayensis is in yellow and L. elongatus samples used by Escobar-Huerta et al. (2015) are in blue. Bootstrap value $\geq 95 \%$ are indicated with a black dot. Numbers in parentheses indicate the amount of collapsed sequences. Scale shows the number of changes per site.
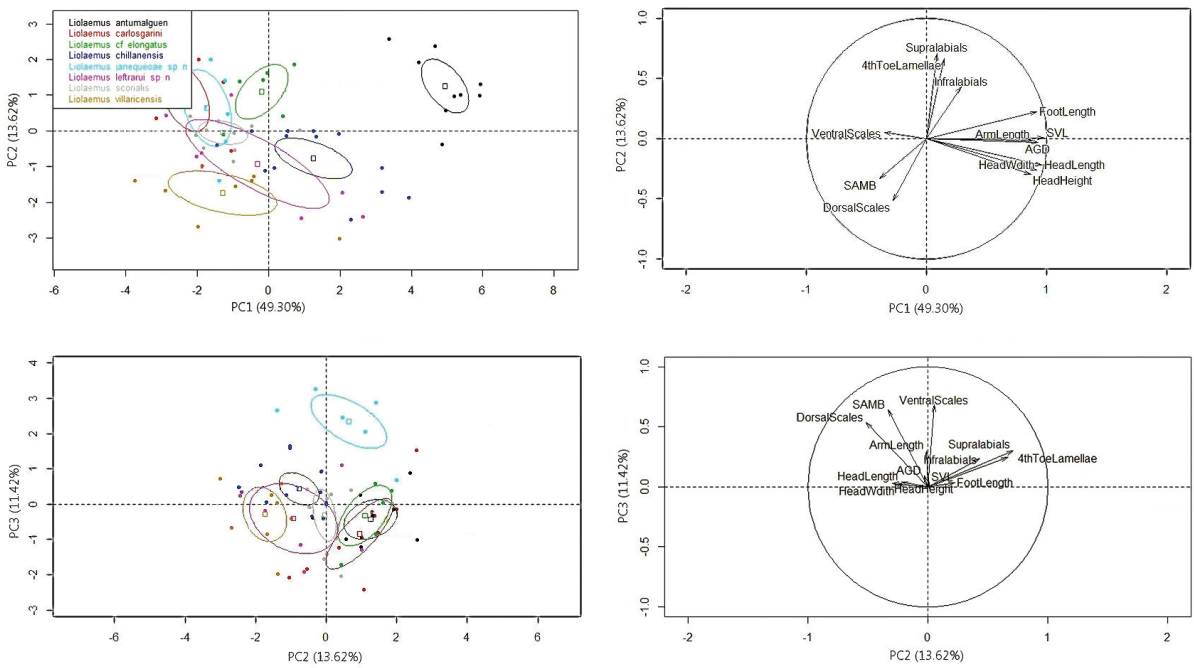

Figure 3. Principal Component Analysis (PCA) results. On the left, ellipses representing the $95 \%$ confidence interval around the centroid for each species. Axis correspond to the percentage of the total variance that each PC explains. On the right, contribution of each variable to the construction of the axes. 
pers. comm.). Additionally, the morphological and coloration differences between the second species described here and L. villaricensis (its sister species), and the uncorrected pairwise difference between them justify the description of this as a new species.

\section{Liolaemus janequeoae sp. $\mathbf{n}$.}

http://zoobank.org/35D080AB-AD1F-4ED5-99E5-CEF925C539FD

Figure 4

Proposed standard English name: Janequeo's Lizard

Proposed standard Spanish name: Lagarto de Janequeo

Holotype. SSUC Re 712 (Fig. 4). Male collected at Laguna Verde $\left(38^{\circ} 12^{\prime} \mathrm{S}-71^{\circ} 44^{\prime} \mathrm{W}\right.$, 1397 masl), approximately $13.5 \mathrm{~km} \mathrm{NW}$ of the summit of the Tolhuaca Volcano, Araucanía Region, Chile. Collected by J. Troncoso-Palacios and Edvin Riveros-Riffo. January 15, 2016.

Paratypes. SSUC Re 713-14. Two females (Fig. 4). Same data as the holotype. SSUC Re 715. Female. Collected at the locality of the holotype by Edvin RiverosRiffo. February 18, 2015. SSUC Re 649-51, three females. Collected at the locality of the holotype by J. Troncoso-Palacios, F. Urra and H. Díaz. January 5, 2014 (Fig. 4).

Diagnosis. Liolaemus janequeoae belongs to the L. elongatus clade. This species is characterized by 1) small size (maximum snout vent length $=69.6 \mathrm{~mm}$ ), 2) lack of dorsal pattern, 3) high number of midbody scales (82-98), 4) precloacal pores present in males, and 5) absence of dark rings on the tail. We provide a differential diagnosis with regards to all species currently considered to be members of this clade, plus $L$. scorialis Troncoso-Palacios Díaz, Esquerré \& Urra, 2015, the assignment of which is under study, but probably is related to the L. elongatus clade (Troncoso-Palacios et al. 2015). Table 1 summarizes some of the diagnostic traits. Based on seven specimens.

Liolaemus janequeoae is closely related to L. elongatus. However, L. janequeoae is smaller (maximum SVL $=69.6 \mathrm{~mm}, \mathrm{n}=7$ adults, vs. $\max . \mathrm{SVL}=94.7 \mathrm{~mm}$ ) and has more midbody scales (82-98 vs. 68-87) than L. elongatus from Argentina (Table 1). Dorsal color pattern in $L$. elongatus is highly variable from vertebral and lateral dark bands to complete melanism, whereas $L$. janequeoae never has black spots (only small black dots in one female). Interestingly, SVL of $L$. cf. elongatus from Llaima, Chile $(\mathrm{SVL}=68.4 \pm 2.9 \mathrm{~mm})$, is not significantly different compared with the SVL of $L$. janequeoae (SVL $=65.3 \pm 3.4 \mathrm{~mm}$ ); but head height is lower in L. janequeoae than in L. cf. elongatus $(6.8 \pm 0.5 \mathrm{~mm}$ vs $8.3 \pm 0.7 \mathrm{~mm})(\mathrm{t}=-4.6, \mathrm{DF}=11, \mathrm{P}<0.01)$; the head is wider in $L$. cf. elongatus than in $L$. janequeoae $(12.7 \pm 0.9 \mathrm{~mm}$ vs $11.0 \pm 0.4 \mathrm{~mm})$ (Mann-Whitney $\mathrm{U}=0.001, \mathrm{P}<0.01) ; L$. janequeoae has more midbody scales than $L$. cf. elongatus (82-98 vs. 76-88) $(\mathrm{t}=3.0, \mathrm{DF}=11, \mathrm{P}<0.05)$, more dorsal scales $(77-89$ vs. 67-73) $(\mathrm{t}=7.7, \mathrm{DF}=11, \mathrm{P}<0.01)$ and more ventral scales $(124-132$ vs. $119-129)$ $(\mathrm{t}=2.5, \mathrm{DF}=11, \mathrm{P}<0.05)$. Additionally, PCA results show that $L$. janequeoae and L. cf. elongatus from Llaima occupy a different region of morphological space, without overlap (Fig. 3). 

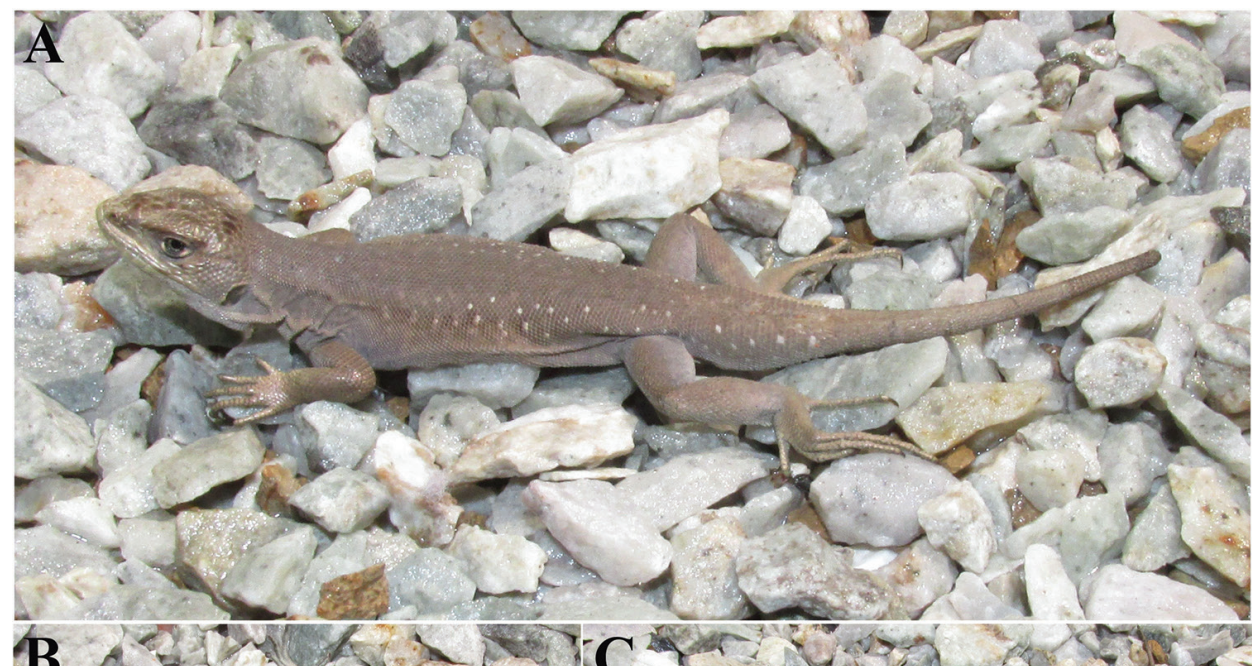

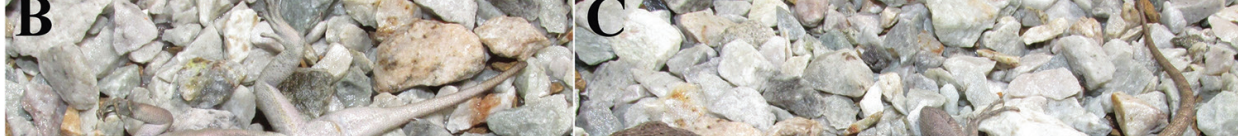

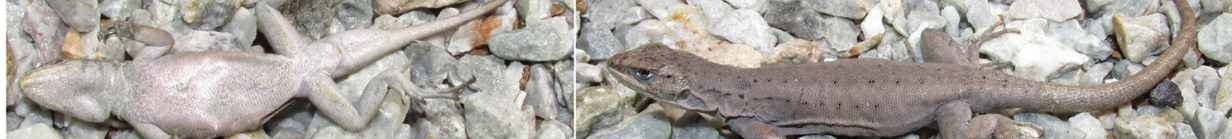

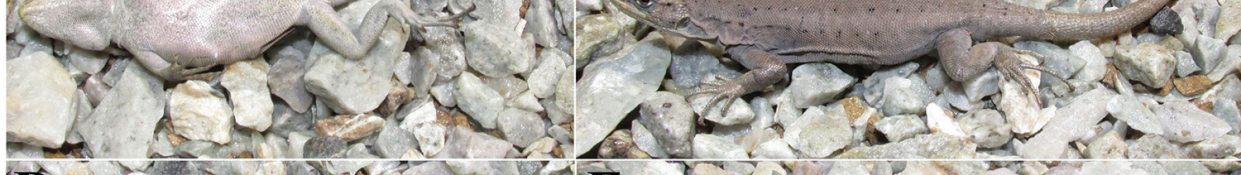

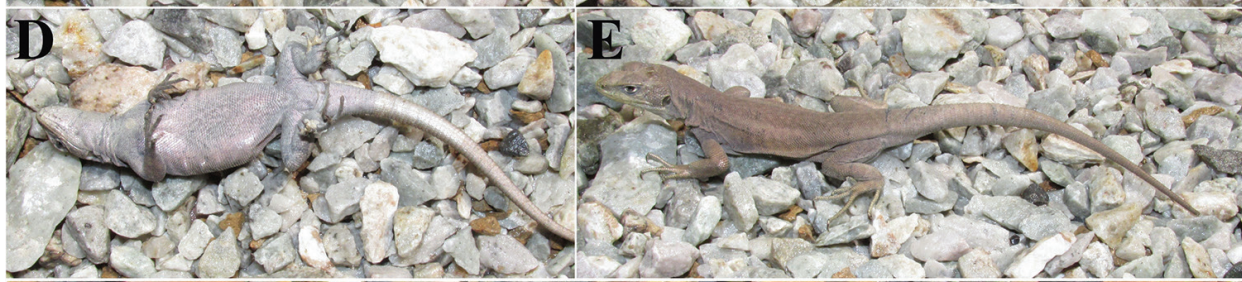

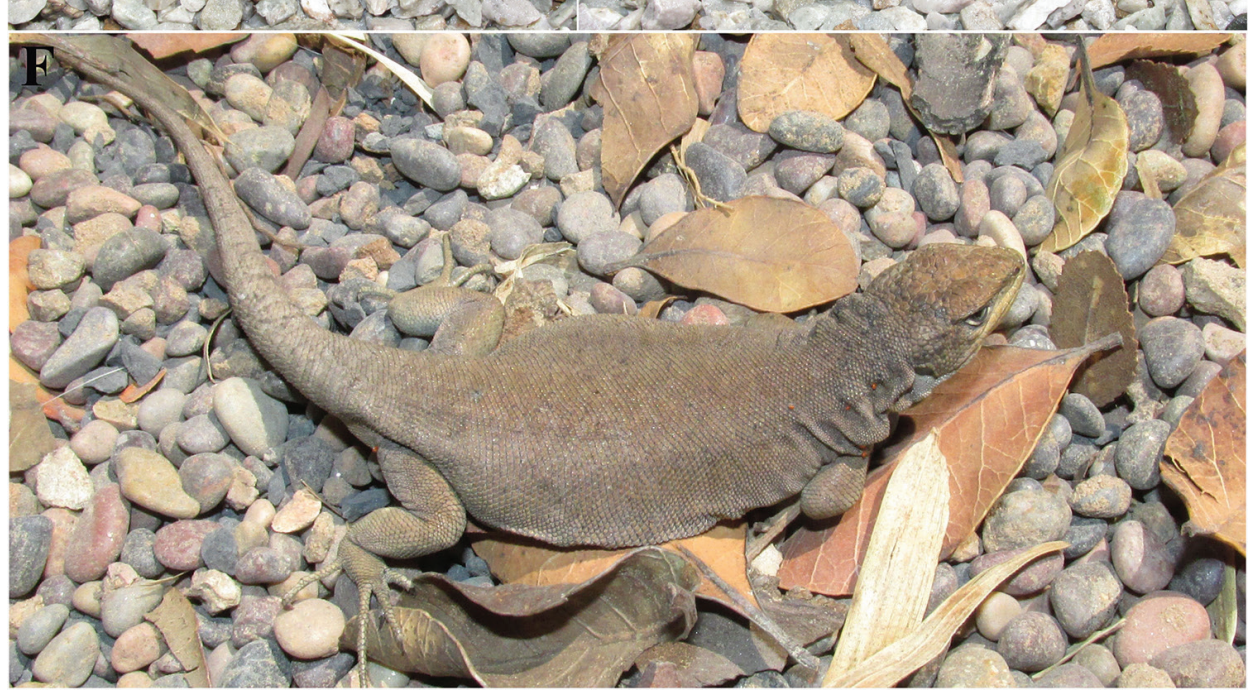

Figure 4. Liolaemus janequeoae sp. n. A and B Holotype, male $\mathbf{C}$ and D Paratype, female with dorsal black dots $\mathbf{E}$ and $\mathbf{F}$ Paratypes, typical females. 


\begin{tabular}{|c|c|c|c|c|c|c|c|c|c|c|c|}
\hline 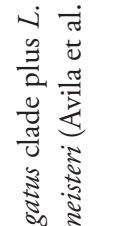 & 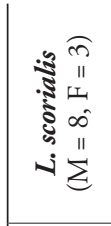 & $\grave{\sigma}$ & 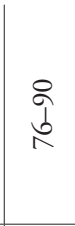 & $\begin{array}{l}\vec{n} \\
\mathfrak{n} \\
n \\
= \\
\vdots\end{array}$ & 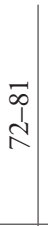 & 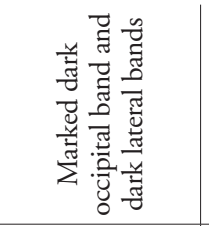 & $\begin{array}{l}\overrightarrow{\tilde{U}^{2}} \\
\text { 完 }\end{array}$ & 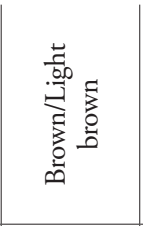 & 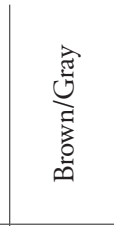 & 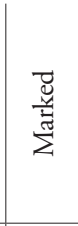 & $y_{m}^{+1}$ \\
\hline 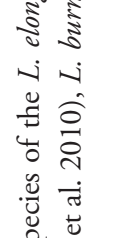 & 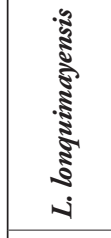 & $\hat{\sigma}$ & $n$. & $\sim$. & $\sim$ & 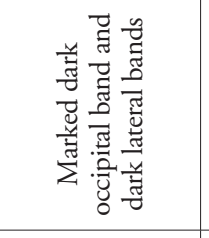 & 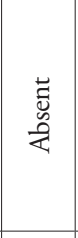 & ڤే & 空 & 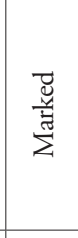 & 0 \\
\hline 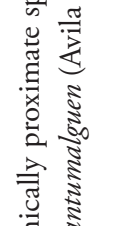 & 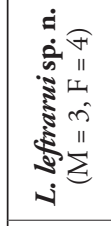 & $\underset{\infty}{\infty}$ & $\begin{array}{l}\infty \\
\infty \\
1 \\
\infty\end{array}$ & 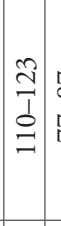 & $\begin{array}{l}\hat{\infty} \\
\hat{N} \\
\hat{N}\end{array}$ & 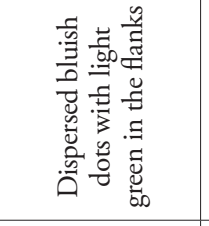 & 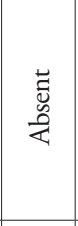 & ڤ్ & 萨 & 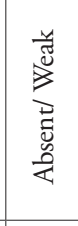 & 0 \\
\hline 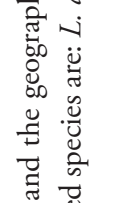 & 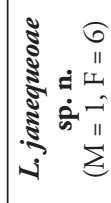 & $\begin{array}{c}0 \\
\hat{\sigma}\end{array}$ & $\begin{array}{l}\stackrel{\infty}{\Im} \\
\underset{\infty}{\sim}\end{array}$ & 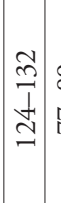 & $\begin{array}{c}2 \\
\infty \\
\hat{\alpha}\end{array}$ & 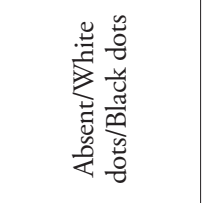 & $\begin{array}{l}\overrightarrow{\tilde{U}} \\
\text { 足 }\end{array}$ & 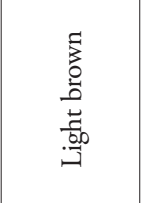 & 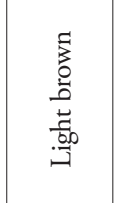 & 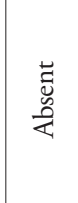 & $m$ \\
\hline 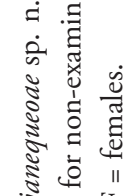 & 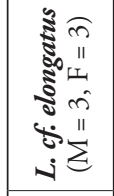 & $\stackrel{m}{\sim}$ & $\begin{array}{l}\infty \\
\infty \\
b \\
\end{array}$ & 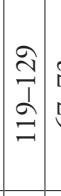 & $\begin{array}{r}\hat{1} \\
\hat{\sigma}\end{array}$ & 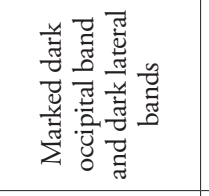 & 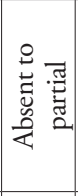 & 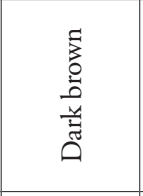 & 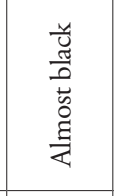 & 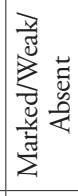 & $\stackrel{n}{\imath}$ \\
\hline 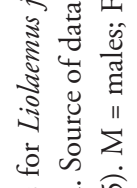 & 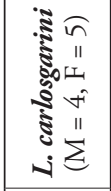 & $\begin{array}{l}\infty \\
\dot{0} \\
0 \\
0\end{array}$ & 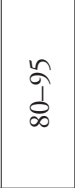 & $\begin{array}{c}\stackrel{d}{d} \\
\stackrel{1}{J} \\
\stackrel{1}{J} \\
\beth\end{array}$ & $\begin{array}{l}\curvearrowright \\
\infty \\
1 \\
0 \\
0\end{array}$ & 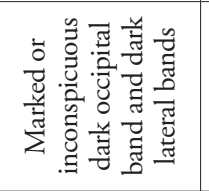 & $\begin{array}{l}\text { 苟 } \\
\text { 荌 }\end{array}$ & 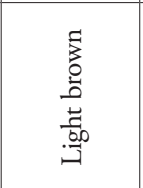 & 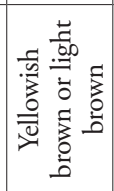 & 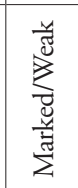 & $\tilde{l}$ \\
\hline 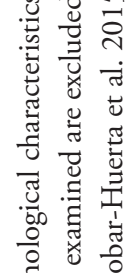 & 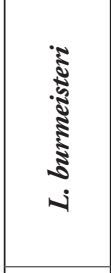 & $\begin{array}{c}\sim \\
\infty \\
\infty\end{array}$ & $\begin{array}{l}\vec{\infty} \\
1 \\
1\end{array}$ & $\begin{array}{l}0 \\
\overline{1} \\
\alpha \\
\sigma\end{array}$ & 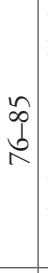 & 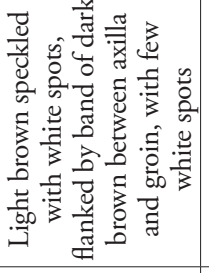 & $\begin{array}{l}\text { 苟 } \\
\text { 荌 }\end{array}$ & 莺 & 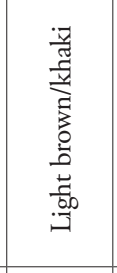 & $\frac{y}{5}$ & $\tilde{\imath}$ \\
\hline 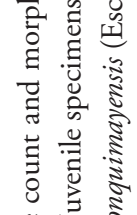 & 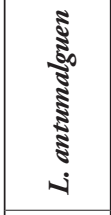 & $\begin{array}{l}\infty \\
\stackrel{0}{0} \\
0\end{array}$ & $\begin{array}{l}\text { Do } \\
\stackrel{N}{N} \\
\end{array}$ & 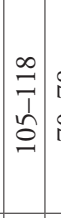 & $\begin{array}{l} \\
\infty \\
\stackrel{1}{1} \\
\stackrel{1}{1}\end{array}$ & 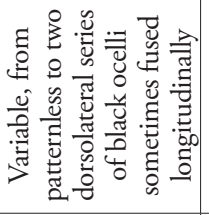 & 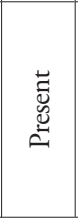 & 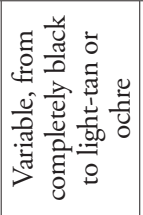 & 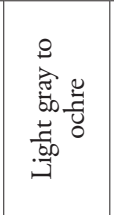 & $\begin{array}{l}\overrightarrow{\tilde{U}} \\
\text { 峖 }\end{array}$ & $\stackrel{+1}{m}$ \\
\hline 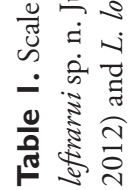 & & 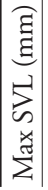 & 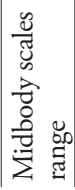 & 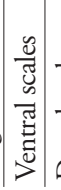 & 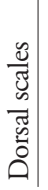 & 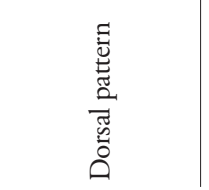 & 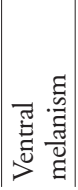 & 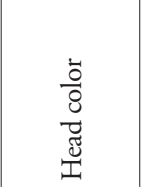 & 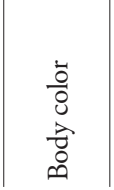 & 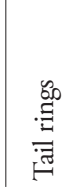 & 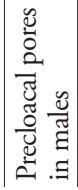 \\
\hline
\end{tabular}


Liolaemus janequeoae is smaller ( $\mathrm{SVL}=65.3 \pm 3.4 \mathrm{~mm}$ ) than L. antumalguen (SVL $=95.0 \pm 6.2 \mathrm{~mm})(\mathrm{t}=-11.3, \mathrm{DF}=14, \mathrm{P}<0.01)$; has a shorter axilla-groin distance $(27.8 \pm 2.9 \mathrm{~mm}$ vs $43.0 \pm 4.4 \mathrm{~mm})$ (Mann-Whitney $\mathrm{U}, \mathrm{P}<0.01)$; a shorter arm length $(24.7 \pm 2.3 \mathrm{~mm}$ vs $28.4 \pm 0.7 \mathrm{~mm})(\mathrm{t}=-4.5, \mathrm{DF}=14, \mathrm{P}<0.01)$; a lower head height $(6.8 \pm 0.5 \mathrm{~mm}$ vs $10.0 \pm 0.6 \mathrm{~mm})(\mathrm{t}=-11.2, \mathrm{DF}=14, \mathrm{P}<0.01)$; a narrower head $(11.0 \pm 0.4 \mathrm{~mm}$ vs $16.6 \pm 0.8 \mathrm{~mm})(\mathrm{t}=-17.2, \mathrm{DF}=14, \mathrm{P}<0.01)$; and has shorter foot length ( $19.4 \pm 1.4 \mathrm{~mm}$ vs $28.5 \pm 1.2 \mathrm{~mm}$ ) (Mann-Whitney $\mathrm{U}, \mathrm{P}<0.01)$; whereas L. janequeoae has more midbody scales than $L$. antumalguen $(\mathrm{t}=6.2, \mathrm{DF}=14, \mathrm{P}<$ 0.01 , Table 1$)$, more dorsal scales $(t=7.6, \mathrm{DF}=14, \mathrm{P}<0.01$, Table 1$)$ and more ventral scales $(\mathrm{t}=8.2, \mathrm{DF}=14, \mathrm{P}<0.01$, Table 1$)$. Moreover, $L$. antumalguen has a very variable dorsal pattern of black spots to almost complete melanism, whereas $L$. janequeoae never has black spots (only small black dots in one female). Additionally, PCA results show that both species occupy a different region of morphological space, without overlap (Fig. 3).

Liolaemus carlosgarini, L. scorialis and L. lonquimayensis have dark lateral and vertebral bands, features that distinguishes these from $L$. janequeoae. Additionally, $L$. janequeoae is larger than $L$. carlosgarini $(\mathrm{SVL}=65.3 \pm 3.4 \mathrm{~mm}$ vs $\mathrm{SVL}=60.2 \pm 5.1$ $\mathrm{mm})(\mathrm{t}=2.4, \mathrm{DF}=22, \mathrm{P}<0.05) ; L$. janequeoae has a larger axilla-groin length than L. carlosgarini $(27.8 \pm 2.9 \mathrm{~mm}$ vs $24.8 \pm 2.9 \mathrm{~mm})(\mathrm{t}=2.3, \mathrm{DF}=22, \mathrm{P}<0.05) ; L$. janequeoae has longer arms than $L$. carlosgarini $(24.7 \pm 2.3 \mathrm{~mm}$ vs $21.8 \pm 1.8 \mathrm{~mm})(\mathrm{t}=$ $3.4, \mathrm{DF}=22, \mathrm{P}<0.01) ;$ L janequeoae has more dorsal scales than $L$. carlosgarini $(\mathrm{t}=$ $4.5, \mathrm{DF}=14, \mathrm{P}<0.01$, Table 1$)$ and more ventral scales $(\mathrm{t}=6.8, \mathrm{DF}=14, \mathrm{P}<0.01$, Table 1); whereas L. lonquimayensis has larger axilla-groin length $(34.9 \pm 1.7 \mathrm{~mm})$ than L. janequeoae (Mann-Whitney $\mathrm{U}, \mathrm{P}<0.05)$; L. lonquimayensis has a greater head height than $L$. janequeoae $(8.3 \pm 0.1 \mathrm{~mm}$ vs $6.8 \pm 0.5 \mathrm{~mm})(\mathrm{t}=-4.8, \mathrm{DF}=8, \mathrm{P}<0.01)$; whereas $L$. scorialis has the head wider than $L$. janequeoae $(11.9 \pm 0.6 \mathrm{~mm}$ vs $11.0 \pm$ $0.4 \mathrm{~mm})(\mathrm{t}=-3.1, \mathrm{DF}=16, \mathrm{P}<0.01) ; L$. janequeoae has more midbody scales than $L$. scorialis $(\mathrm{t}=3.6, \mathrm{DF}=16, \mathrm{P}<0.01$, Table 1$)$ and more dorsal scales $(\mathrm{t}=4.8, \mathrm{DF}=17$, $\mathrm{P}<0.01$, Table 1 ). Additionally, PCA results show that $L$. janequeoae does not overlap in the morphological space with $L$. carlosgarini and $L$. scorialis when ellipses are generated with the second and third PCs (Fig. 3).

Liolaemus janequeoae is smaller (max. SVL $=69.6 \mathrm{~mm}$ ) than L. shitan (max. SVL $=98.3 \mathrm{~mm}$ ) and has more midbody scales (82-98 vs. $72-85)$. Dorsal color pattern in $L$. shitan is black, whereas only one female of our sample of $L$. janequeoae has small dorsal black dots.

Liolaemus janequeoae is smaller (max. SVL $=69.6 \mathrm{~mm}$ ) than L. choique (max. SVL $=90.7 \mathrm{~mm}$ ). Moreover, $L$. choique has a very variable dorsal pattern of black spots to almost complete melanism, whereas $L$. janequeoae never has black spots (only small black dots in one female).

Liolaemus janequeoae is smaller than L. crandalli (max. SVL $=69.6 \mathrm{~mm}$ vs max. $S V L=93.4 \mathrm{~mm}$ ). Moreover, $L$. crandalli has dark lateral and vertebral bands with ringed tail, whereas all of these features are completely absent in $L$. janequeoae. According to Avila et al. (2015), L. crandalli is the sister taxon of the pair L. smang $+L$. 
choique, whereas in our phylogeny L. janequeoae is not closely related to L. smang or L. choique.

Liolaemus janequeoae is smaller than L. burmeisteri $(\max . S V L=69.6 \mathrm{~mm}$ vs $\max$. $\mathrm{SVL}=85.2 \mathrm{~mm})$ and has more midbody (82-98 vs. 70-81) and ventral scales (124132 vs. 99-110). Moreover, L. burmeisteri has dark lateral bands.

Liolaemus janequeoae has more midbody scales than L. smaug (82-98 vs 73-80). Moreover, $L$. smang has dark lateral and vertebral band. In our phylogeny $L$. janequeoae and $L$. smang are not sister taxa.

Description of holotype. Adult male. SVL: $59.1 \mathrm{~mm}$. Tail length: $42.0 \mathrm{~mm}$ (autotomized). Axilla-groin length: $21.8 \mathrm{~mm}$. Head length: $13.1 \mathrm{~mm}$. Head width (distance between the two ear openings): $10.5 \mathrm{~mm}$. Head height (at the level of ear openings): $6.1 \mathrm{~mm}$. Forelimb length: $21.1 \mathrm{~mm}$. Hindlimb length: $36.0 \mathrm{~mm}$. Foot length: $18.6 \mathrm{~mm}$. Hand length: $9.8 \mathrm{~mm}$. Rostral scale wider $(2.36 \mathrm{~mm})$ than high $(0.8 \mathrm{~mm})$. Subocular length: $4.2 \mathrm{~mm}$. Fifth supralabial length: $1.6 \mathrm{~mm}$. Neck width: $9.4 \mathrm{~mm}$. Interorbital distance: $4.5 \mathrm{~mm}$. Internasal distance: $1.5 \mathrm{~mm}$. Body width: $13.7 \mathrm{~mm}$. Meatus width: $1.4 \mathrm{~mm}$. Meatus height: $2.1 \mathrm{~mm}$.

Two postrostrals. Four internasals. Hexagonal interparietal scale, with a central, small, and whitish "parietal eye" in the center. Interparietal smaller than the parietals, surrounded by other nine scales; ten scales between interparietal scale and rostral; seventeen scales between occiput and rostral (Hellmich Index); orbital semicircles are interrupted by one supraocular scales in both sides, but the rest is formed by ten scales on each side; 6-7 supraoculars (left-right); six superciliary scales. Frontal area is divided into three scales (one posterior, one middle and one anterior). Two scales between the nasal and the canthal. Preocular separated from the lorilabials by a single loreal scale. Nasal separated from rostral by one scale, surrounded by seven scales. One row of lorilabials between the supralabials and the subocular; seven supralabials, the fifth is curved upward without contacting the subocular; six infralabial scales. Mental scale is pentagonal, in contact with four scales; four pairs of postmental shields, the second is separated by two scales. Temporal scales are subimbricated and smooth or slightly keeled. Eleven temporal scales between the level of superciliary scales and the commissure of the mouth. Two projecting scales on the anterior edge of the ear, which do not cover the auditory meatus. Auricular scale is wide and restricted to the upper third of the meatus; 44 gulars between the auditory meatuses. Antehumeral fold and "Y" shaped lateral neck fold. Developed dorsolateral fold. Midbody scales: 94. Dorsal scales are rhomboidal, slightly keeled, without mucrons, subimbricate and with interstitial granules. Dorsal scales are similar in size than ventral ones. Dorsal scales: 89. Ventral scales are rhomboidal, smooth, imbricate, and without interstitial granules. Ventral scales: 124. Three precloacal pores. Hemipenial bulges are evident. The suprafemoral scales are lanceolate, imbricate, and slightly keeled. Infrafemoral scales are lanceolate to rounded, smooth, and imbricate. Scales of the dorsal surface of the forearm are lanceolate to rounded, imbricate, and slightly keeled or smooth. Scales of the ventral surface of the forearm are rounded, smooth, and subimbricate. The dorsal scales of the first third of the tail are rhomboidal to lanceolate, subimbricate or juxtaposed, keeled and with inter- 
stitial granules. The ventral scales of the tail vary from rhomboidal to triangular, and are imbricate and smooth. Lamellae of the fingers: I: 10, II: 14, III: 22, IV: 24 and V: 15. Lamellae of the toes: I: 11, II: 16, III: 22, IV: 32 and V: 19.

Coloration in life. Light brown head, with dark brown spots in the parietal area and in the posterior nasal area. The snout is olive. Temporal area is light brown. Subocular area and cheeks are slightly lighter than temporal area. The subocular is immaculate. Background color of the dorsum, limbs, and tail is light brown. The vertebral zone of the dorsum is slightly darker than rest, but without forming an occipital stripe. The only dorsal design is a series of white dots, formed by 1-3 white scales, running from the posterior half of the trunk to the first third of the tail. The tail is immaculate. Ventrally, the throat, belly, limbs and the tail are whitish pearly. Thighs and cloaca have a little yellowish coloration. Precloacal pores are orange.

Variation. Despite four field campaigns, no additional males were found. Variation in measures refer to the six female paratypes: SVL: 66.2-69.6 mm. Axilla-groin distance: $27.4-30.2 \mathrm{~mm}$. Head length: $13.5-15.1 \mathrm{~mm}$. Head width: $10.7-11.4 \mathrm{~mm}$. Head height: $6.4-7.6 \mathrm{~mm}$. Foot length: 18.0-21.5 mm. Leg length: 36.5-44.7 mm. Hand length: 9.4-11.7 mm. Arm length: 21.1-26.7 mm. Tail length: 84-110 ( $\mathrm{n}=3$; autotomized in the rest). Relation tail length/SVL $=1.2-1.7$. Although more data on males are required, there is no sexual size dimorphism in the Liolaemus elongatus clade species (Avila et al. 2012).

Scale number variation in Liolaemus janequeoae (all specimens) is as follows. Midbody scales: 82-98 (91.6 \pm 5.5$)$. Dorsal scales: $77-89(85.0 \pm 4.2)$. Ventral scales 124-132 (128.6 \pm 3.5$)$. Fourth finger lamellae: 22-24 (23.5 \pm 0.8$)$. Fourth toe lamellae: $28-32(29.5 \pm 1.4)$. Supralabial scales: 6-8 (7.4 \pm 0.8$)$. Infralabial scales: $5-6$ (5.3 $\pm 0.5)$. Interparietal scale is pentagonal or hexagonal, bordered by $5-9$ scales $(6.6 \pm 1.7)$. The interparietal is smaller than the parietals. The nasal is in contact with the rostral in $28.6 \%$ of specimens.

Females have a very similar color pattern to the male holotype but without dorsal white dots or yellowish coloration on the thighs and cloaca. One female has four series of black dots (formed by 1-3 black scales) on the dorsum: two on the paravertebral fields (running from the head to the first third of the tail) and two on the dorsolateral area (running from the head to the middle of the trunk).

Etymology. This species is named after Janequeo, a prominent Lonko (tribal chief) of Mapuche-Pehuenche origins. She fought against colonial Spaniards in the Arauco war, carried out mainly in the Araucanía Region where Liolaemus janequeoae was discovered. It is believed that she became involved in the war after her partner (Lonko Hueputan) was captured and tortured to death. She played a leading role in the Battle of Fort Puchunqui, then retreating to Villarrica, where she disappeared.

Distribution and natural history. Only known from the type locality at Laguna Verde $\left(38^{\circ} 12^{\prime} \mathrm{S}-71^{\circ} 44^{\prime} \mathrm{W}\right)$, approximately $13.5 \mathrm{~km}$ NW of the summit of the Tolhuaca volcano, Araucanía Region, Chile (Fig. 5).

At Laguna Verde, Liolaemus janequeoae was found between 1336-1397 masl. It inhabits the deciduous highland Andean forest (Gajardo 1994), consisting of Arau- 


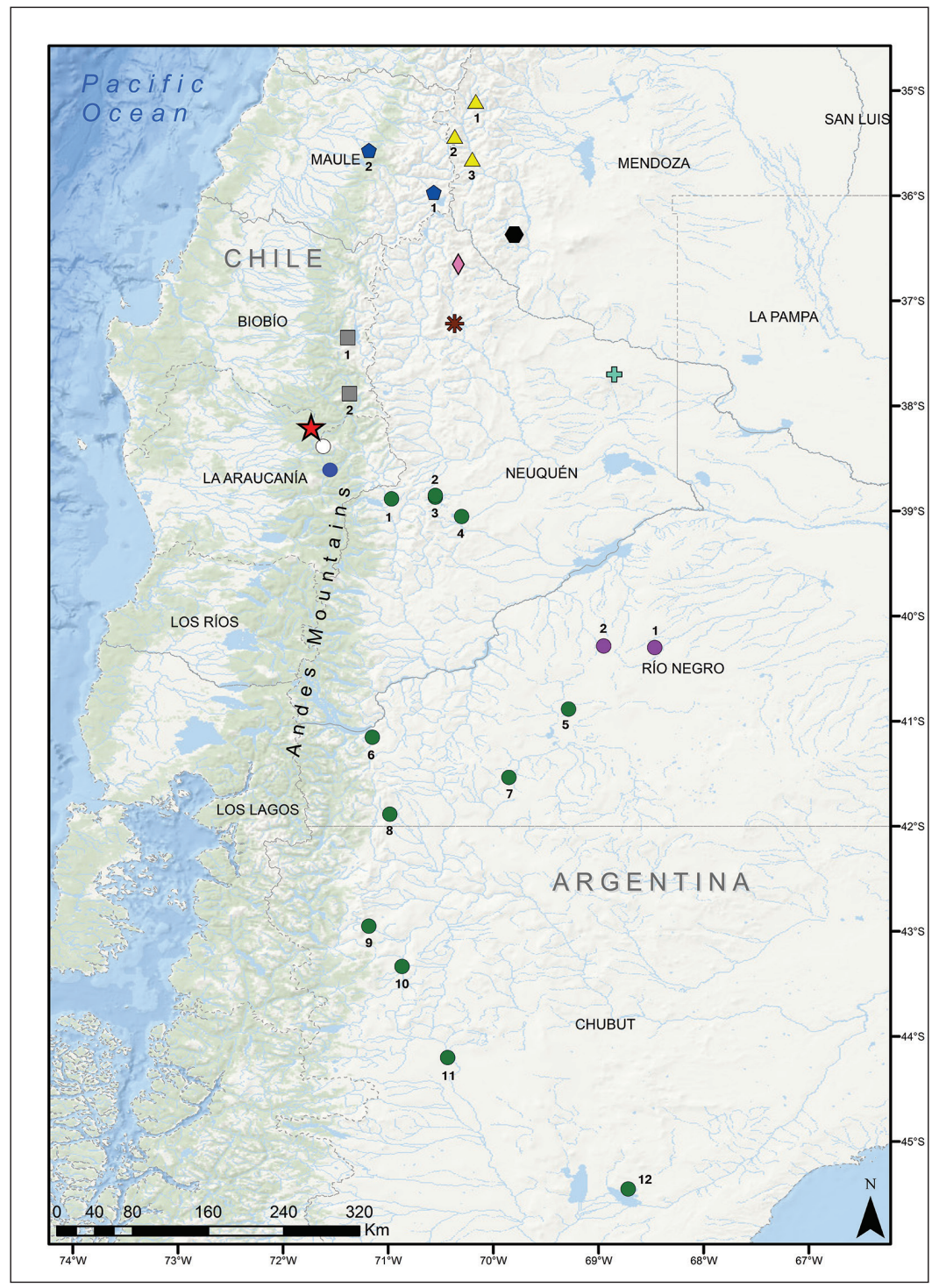

Figure 5. Distribution map for Liolaemus janequeoae sp. n. with geographically proximate species of the $L$. elongatus clade. In the case of $L$. elongatus a sample for each locality was included in the phylogeny. Red star: L. janequeoae sp. n., Laguna Verde. Yellow triangles: L. smaug $(1=$ near Las Leñas, $2=$ between Las Loicas and Peteroa Volcano, 3= near Las Loicas). Blue pentagon: L. carlosgarini (1= Maule Lagoon, 2= Lircay). Black hexagon: L. choique (Paso el Choique). Pink diamond: L. antumalguen (Domuyo Volcano). Brown asterisk: L. burmeisteri (Caepe Malal). Green cross: L. crandalli (Auca Mahuida Volcano). Gray squares: L. scorialis (1 = Laja Lagoon, 2= La Mula Lagoon). White circle: L. lonquimayensis (Lonquimay Volcano). Pink circles: L. shitan ( $1=$ Estancia Piedras Blancas, type locality and $2=$ near Antonio del Cuy). Blue circle: L. cf. elongatus (Llaima Volcano). Green circles: L. elongatus (1= Pampa de Lonco Luan, 2= Primeros Pinos, 3= Portal La Atravesada, 4= Laguna Blanca, 5= near Ingeniero Jacobacci, 6= San Carlos de Bariloche, 7= Ojo de Agua, 8= El Maiten, 9= Esquel, 10= Tecka, 11= Gobernador Costa and $12=$ Los Manantiales). 
caria araucana and Nothofagus dombeyi (1397 masl). The shrubs are represented by Chusquea culeou, Desfontainia spinosa, Drimys andina and Pseudopanax laetevirens. At lower altitudes (1336 masl), the vegetation was dominated by $A$. araucana and $N$. pumilio, with the presence of Azara alpine, C. culeou, Colletia hystrix, Lomatia hirsuta, Maytenus disticha, Myrcengenia chrysocarpa and Pernettya myrtilloides. At lower altitudes where there are no Araucaria araucana, L. janequeoae was not found. It is a diurnal lizard of apparently low abundance. It was seen on rocks and climbing in trees.

Liolaemus janequeoae was found in syntopy with L. septentrionalis Pincheira-Donoso and Núnez, 2005; L. tenuis (Duméril and Bibron, 1837); Pristidactylus torquatus (Philippi, 1861) and the second new species described below. In this zone, it was also recorded the presence of Tachymenis chilensis (Schlegel, 1837).

The intestinal content of one specimen (paratype) was examined and remnants of insects and several nematodes were found. At the date of capture (January 5) two females had two and three embryos each. All other females have only several small oocytes.

\section{Liolaemus leftrarui sp. $\mathrm{n}$.}

http://zoobank.org/71CE0862-31F7-4ADD-B977-F00479198873

Figure 6

Proposed standard English name: Leftraru`s Lizard

Proposed standard Spanish name: Lagarto de Leftraru

Holotype. SSUC Re 646 (Fig. 6a, b). Male collected at Laguna Verde $\left(38^{\circ} 12^{\prime} S\right.$ $71^{\circ} 44^{\prime} \mathrm{W}, 1405$ masl), approximately $13.5 \mathrm{~km} \mathrm{NW}$ of the summit of the Tolhuaca volcano, Araucanía Region, Chile. Collected by J. Troncoso-Palacios, F. Urra and H. Díaz. January 5, 2014.

Paratypes. SSUC Re 647-48, 716 (Fig. 6). Three females. Same data as the holotype. SSUC Re 732-734. Two males and one female. Near Lagunillas, Araucanía Region, Chile $\left(38^{\circ} 12^{\prime} \mathrm{S}-71^{\circ} 46^{\prime} \mathrm{W}, 1483\right.$ masl), approximately $4 \mathrm{~km} \mathrm{NW}$ from the type locality. Collected by J. Troncoso-Palacios \& E. Villarroel. September, 2016.

Diagnosis. Liolaemus leftrarui is closely related to L. villaricensis. This species is characterized by 1) lack of precloacal pores in either sex, 2) large size Liolaemus (max. SVL $=81.8 \mathrm{~mm}$ ), 3) high amount of midbody scales (80-88), 4) light blue dots on the dorsum, and 5) absence of ventral melanism. We provide a diagnosis in regards to L. villaricensis, plus four unrelated species that occur geographically near to $L$. leftrarui and that also feature the absence of precloacal pores. Based on seven specimens.

Liolaemus leftrarui has more dorsal scales than L. villaricensis (77-87 vs. 80-89) (t $=-2.5, \mathrm{DF}=11, \mathrm{P}<0.05)$. Moreover, $L$. villaricensis has a marked lateral black band and a fragmented vertebral stripe, whereas in L. leftrarui these two color features are inconspicuous or less marked than in L. villaricensis. Liolaemus villaricensis has no light blue dots, which are in all specimens of $L$. leftrarui. Finally, although they are sister species, the average uncorrected pairwise distance between the two taxa is $7.3 \%$, more than double that value proposed for identification of candidate species in Liolaemus. 


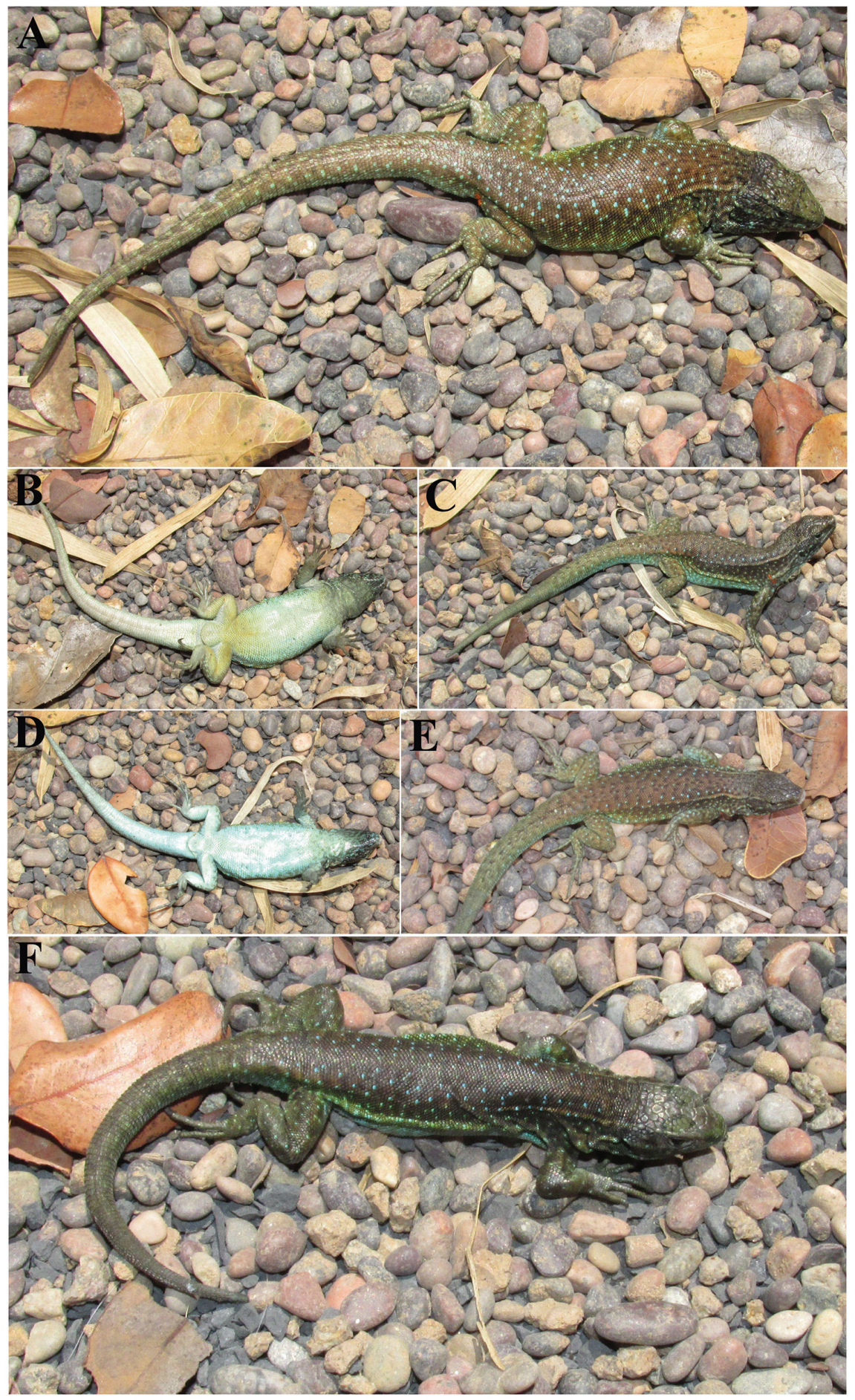

Figure 6. Liolaemus leftrarui sp. n. A and B Holotype, male C Dorsal and D ventral view of Paratype, female $\mathbf{E}$ and $\mathbf{F}$ Paratypes, females. 
Additionally, PCA results show that both species only marginally overlap in morphological space when ellipses are generated with the two first PCs (Fig. 3).

Liolaemus leftrarui is larger ( $\max . \mathrm{SVL}=81.8 \mathrm{~mm}$ ) than L. coeruleus (males SVL $=58.7 \pm 3.2 \mathrm{~mm}$; females SVL $=58.2 \pm 2.8 \mathrm{~mm}$ ) and L. neuquensis (males SVL = $57.4 \pm 3.5 \mathrm{~mm}$; females SVL = $58.2 \pm 1.9 \mathrm{~mm}$ ). Moreover, $L$. coeruleus males feature black ventral color and some $L$. neuquensis males also feature a black ventral color, a feature absent in L. leftrarui. Females of L. coeruleus and L. neuquensis have a brown dorsal color, but females of $L$. leftrarui have a bluish brown dorsal color. Finally, in our phylogeny L. neuquensis is not closely related to L. leftrarui and although we have no molecular data for L. coeruleus, this last species and L. neuquensis are probably conspecific (Avila et al. 2003).

Liolaemus leftrarui has more midbody scales (80-88 vs. 67-81) than L. punmahuida. Dorsal color in L. punmahuida is ochre and this species is patternless, whereas $L$. leftrarui has brown dorsal color with dispersed light blue dots. Liolaemus punmahuida has reddish color around the cloaca, feature absent in L. leftrarui. The species are not closely related according to our phylogeny.

Liolaemus leftrarui differs from L. tregenzai in that this last species features black color on the throat, chest and abdomen of males and gray color on the throat, chest and abdomen of females, features totally absent in L. leftrarui. The species are not closely related according to our phylogeny.

Description of holotype. Adult male. SVL: $81.7 \mathrm{~mm}$. Tail length: $102.9 \mathrm{~mm}$ (not autotomized). Axilla-groin length: $35.4 \mathrm{~mm}$. Head length: $20.1 \mathrm{~mm}$. Head width (distance between the two ear openings): $16.9 \mathrm{~mm}$. Head height (at the level of ear openings): $10.8 \mathrm{~mm}$. Forelimb length: $26.5 \mathrm{~mm}$. Hindlimb length: $46.0 \mathrm{~mm}$. Foot length: $21.8 \mathrm{~mm}$. Hand length: $13.6 \mathrm{~mm}$. Rostral scale wider $(4.3 \mathrm{~mm})$ than high $(1.6$ $\mathrm{mm})$. Subocular length: $5.7 \mathrm{~mm}$. Fourth supralabial length: $3.4 \mathrm{~mm}$. Neck width: $16.2 \mathrm{~mm}$. Interorbital distance: $7.2 \mathrm{~mm}$. Internasal distance: $3.0 \mathrm{~mm}$. Body width: $27.2 \mathrm{~mm}$. Meatus width: $1.0 \mathrm{~mm}$. Meatus height: $3.3 \mathrm{~mm}$.

Two postrostrals. Four internasals. Pentagonal interparietal scale, with a central, small, and whitish "parietal eye" in the center. Interparietal scale is similar in size to parietal one, surrounded by other six scales; seven scales between interparietal scale and rostral; twelve scales between occiput and rostral; orbital semicircle is incomplete in the right side and complete in the left side (formed by 12 scales); 5-4 supraoculars (left-right); five superciliary scales. Frontal area is divided into three scales (two posterior and one anterior). Remarkably, only one scale between the nasal and the canthal. Preocular separated from the lorilabials by a single loreal scale. Nasal in contact with the rostral, surrounded by seven scales. One row of lorilabials between the supralabials and the subocular; six supralabials, the fourth is curved upward without contacting the subocular; five infralabial scales. Mental scale is pentagonal, in contact with four scales; five pairs of postmental shields, the second is separated by two scales. Temporal scales are subimbricate and smooth, very few are slightly keeled. Eight temporal scales between the level of superciliary scales and the level of the commissure of the mouth. Two enlarged projecting scales on the anterior edge of the ear, which do not cover 
the auditory meatus. Auricular scale is wide and restricted to the upper third of the meatus; 42 gulars between the auditory meatuses. Antehumeral fold and "Y" shaped lateral neck fold. Present inconspicuous ventrolateral fold. Midbody scales 86. Dorsal scales are rounded to lanceolate, slightly keeled, without mucrons, imbricate and with some interstitial granules. Dorsal scales are smaller than ventral ones. Dorsal scales 81 . Ventral scales are rhomboidal to rounded, smooth, imbricate, and without interstitial granules. Ventral scales 118. There are no precloacal pores. Hemipenial bulges are evident. The suprafemoral scales are lanceolate, imbricate, and smooth or slightly keeled. Infrafemoral scales are rounded, smooth, and imbricate. Scales of the dorsal surface of the forearm are rounded, imbricate, and slightly keeled or smooth. Scales of the ventral surface of the forearm are rounded, smooth, juxtaposed or subimbricate with interstitial granules. The dorsal scales of the tail are rhomboidal, imbricate, keeled and some with mucrons. The ventral scales of the tail vary from rhomboidal to triangular, and are imbricate and smooth. Lamellae of the fingers: I: 12, II: 14, III: 20, IV: 22 and V: 15. Lamellae of the toes: I: 11, II: 16, III: 21, IV: 27 and V: 18.

Coloration in life. Brown head, with dispersed dark brown spots. Occipital area of the head is dark brown; temporal area is brown with three dark brown stripes and some dispersed light blue scales. Ocular area, snout and cheeks are light green. Subocular scale is light blue with two dark brown vertical lines, one in the middle and other in the anterior edge. Background color of the dorsum is brown. Inconspicuous dorsolateral light brown stripe (two scales of wide) running from the occiput level to the level of the axilla. Dark brown spots dispersed on the dorsum, without forming an occipital band, but forming three lines on the neck; one of which (middle) forms an inconspicuous vertebral stripe on the dorsum. Several light blue dots dispersed on the dorsum (each corresponds to one scale). Inconspicuous dark brown lateral band with dispersed light blue scales. Below lateral band, flanks are yellowish. Limbs are brown with light green and few dispersed light blue scales. Tail is brown with dispersed light green scales and dark brown vertebral line. Ventrally, the throat is dark green, darker towards the tip of the snout. Belly and the tail are light green. Rear portion of belly, cloaca, chest and thighs have a yellowish coloration. Palms are dark brown and soles are light brown.

Variation. Variation in three males (including the holotype): SVL: 76.1-81.8 $\mathrm{mm}$. Axilla-groin distance: 33.2-35.7 mm. Head length: 17.9-20.1 mm. Head width: 14.6-16.9 mm. Head height: $9.3-10.8 \mathrm{~mm}$. Foot length: $20.2-21.8 \mathrm{~mm}$. Leg length: 42.7-46.0 mm. Hand length: $12.0-13.6 \mathrm{~mm}$. Arm length: $26.0-27.3 \mathrm{~mm}$. Tail autotomized in all male paratypes. Variation in four female paratypes is as follows: SVL: 60.5-68.2 mm. Axilla-groin distance: $26.4-30.1 \mathrm{~mm}$. Head length: $13.2-15.0 \mathrm{~mm}$. Head width: 9.7-12.0 mm. Head height: $6.3-7.0 \mathrm{~mm}$. Foot length: $17.4-17.9 \mathrm{~mm}$. Leg length: 32.5-38.2 mm. Hand length: 10.1-11.1 mm. Arm length: 20.5-21.2 $\mathrm{mm}$. Tail autotomized in all females.

Scale number variation in Liolaemus leftrarui (all specimens) is as follows. Midbody scales: $80-88(84.3 \pm 3.5)$. Dorsal scales: $77-87(81.3 \pm 3.6)$. Ventral scales $108-123$ $(115.3 \pm 5.8)$. Fourth finger lamellae: $20-23(21.9 \pm 1.1)$. Fourth toe lamellae: $27-30$ 
(28.1 \pm 1.3$)$. Supralabial scales: $6-7(6.4 \pm 0.5)$. Infralabial scales: $4-5(4.7 \pm 0.5)$. Holotype has only one scale between the nasal and the canthal, but paratypes have two, as usual in the genus Liolaemus. No precloacal pores in the males and no vestigial precloacal pores in the females, which is rare in Liolaemus. Interparietal scale is quadrangular, pentagonal, hexagonal or heptagonal, bordered by $5-7$ scales $(5.7 \pm 0.8)$. The interparietal is similar size or smaller than the parietals. The canthal is in contact with the rostral in all specimens.

Paratype males have similar coloration pattern to the holotype with variation only in shade. Females have similar coloration pattern to the holotype, but with some differences such as: the dark brown color on the occipital area is less marked or absent; the dark brown lateral band (inconspicuous in the holotype) is marked in some females; the dark brown vertebral stripe of the tail is inconspicuous or absent in females; the ventral color is light green or light blue; the throat is reticulated in one female; the yellowish color on the rear portion of belly and the cloaca is less marked or absent in females.

Etymology. This species is named after Leftraru, the most prominent Lonko (tribal chief) of the Mapuche people, who fought against colonial Spaniards in the Arauco war, carried out mainly in the Araucanía Region where we discovered Liolaemus leftrarui. He was captured when he was eleven by Pedro de Valdivia (Governor of the Kingdom of Chile) and became his personal servant. He learned the military strategy of the Spanish and then escaped. Later, he ambushed and killed Valdivia, and won the most remarkable victories over the Spaniards. Finally, he was surrounded and died in battle.

Distribution and natural history. Known from two localities: 1) the type locality at Laguna Verde $\left(38^{\circ} 12^{\prime} \mathrm{S}-71^{\circ} 44^{\prime} \mathrm{W}\right)$, approximately $13.5 \mathrm{~km} \mathrm{NW}$ of the summit of the Tolhuaca volcano, Araucanía Region, Chile (Fig. 7). At Laguna Verde, Liolaemus leftrarui was found between 1336-1397 masl. Vegetation is the same described for the habitat of $L$. janequeoae. At lower altitudes where there are no Araucaria araucana, $L$. leftrarui was not found. 2) Near Lagunillas (38 $12^{\circ} \mathrm{S}-71^{\circ} 46^{\prime} \mathrm{W}, 1483$ masl), approximately $4 \mathrm{~km} \mathrm{NW}$ from Laguna Verde, in the Araucaria araucana forest. It is probable that the distribution of $L$. leftrarui could extend to Lagunillas (1700 masl) but in September (date of collection) this area is covered with snow and no specimens were found. Remarkably, L. janequeoae was not found near Lagunillas. Liolaemus leftrarui is a diurnal lizard of apparently low abundance at both localities. It was seen on rocks and trees (in Laguna Verde), clambering to approximately $5 \mathrm{~m}$ aboveground in trees when threatened. Near Lagunillas it was seen only in fallen trees.

Liolaemus leftrarui was found in syntopy with L. septentrionalis, L. tenuis, L. janequeoae and Pristidactylus torquatus at the type locality. Near Lagunillas it was found in syntopy with L. septentrionalis and L. tenuis. In this zone the presence of Tachymenis chilensis was also recorded.

The intestinal contents of one specimen from the type locality was examined and revealed the remnants of insects. No plant remains were found. One specimen from near Lagunillas had several nematodes in the intestines. The females collected in January had several small oocytes but the female collected in September carried one embryo. 


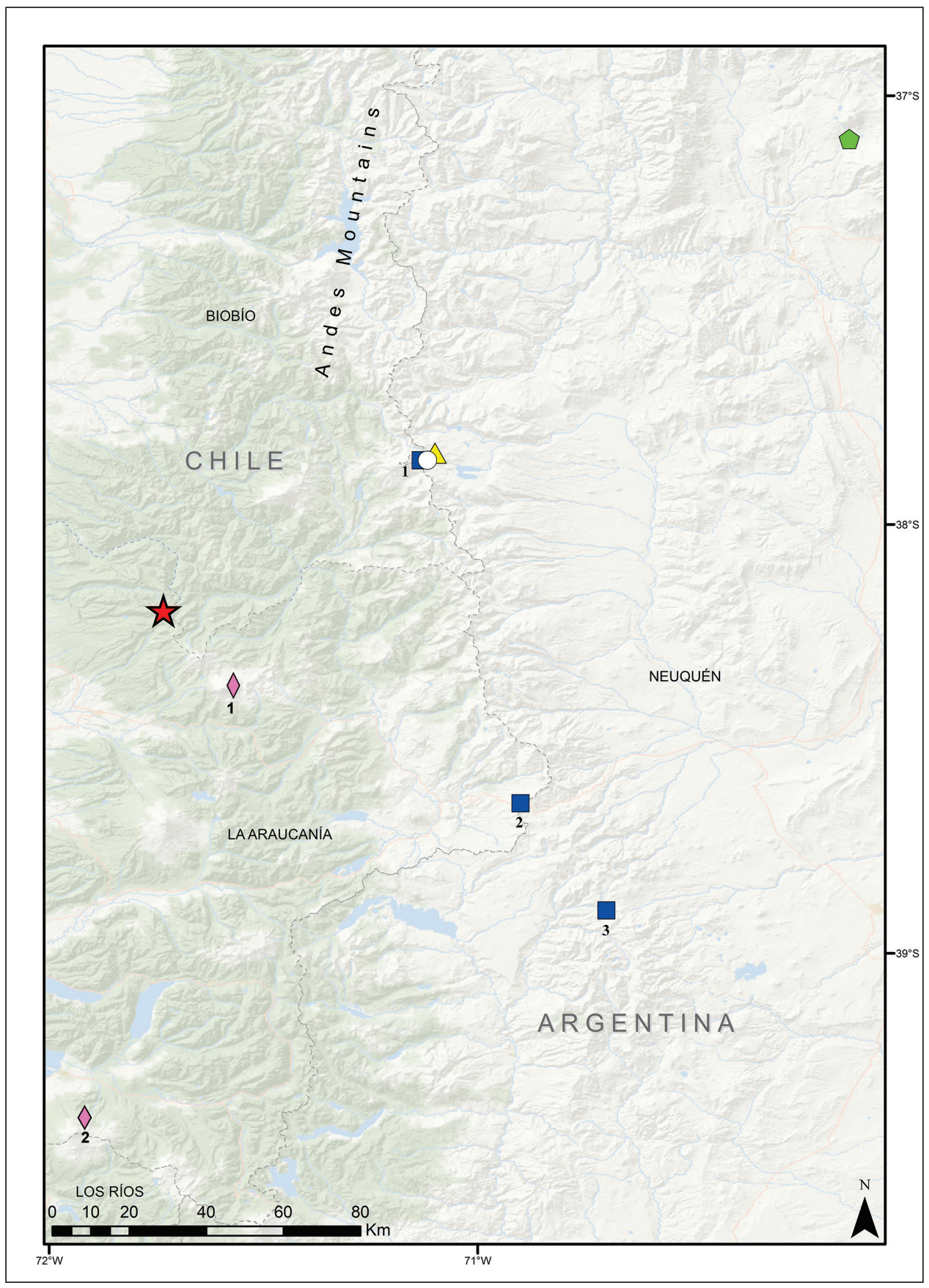

Figure 7. Distribution map for Liolaemus leftrarui sp. n. with closely related L. villaricensis and geographically proximate species that feature a lack of precloacal pores. Red star: L. leftrarui sp. n. (Laguna Verde and Lagunillas). Pink diamond: L. villaricensis ( $1=$ Lonquimay Volcano, $2=$ Villarrica Volcano). Blue squares: $L$. coeruleus ( $1=$ Copahue, $2=$ Pino Hachado, $3=$ Primeros Pinos). White circle: L. tregenzai (Copahue). Yellow triangle: L. neuquensis (Copahue). Green pentagon: L. punmahuida (Tromen Volcano). 


\section{Discussion}

The diversity of the Chilean members of the Liolaemus elongatus-kriegi complex has been largely underestimated. Recent expeditions to seldom explored highlands and the revision of the taxonomic status of some populations has led to the description of several new species (Escobar-Huerta et al. 2015, Esquerré et al. 2013, 2014, Núñez 2007, Troncoso-Palacios et al. 2015). In fact, it has been thought that Chilean species of the L. elongatus-kriegi complex have a small distribution in central Chile (Morando et al. 2003), but currently it is known that this group of lizards is widely distributed in central and southern Chile, and it is also probable that some populations under study could be described as new species in the future (Troncoso-Palacios unpublished data).

The new species, Liolaemus janequeoae, was found to be member of the L. elongatus clade and the sister species of the clade formed of $L$. elongatus $+L$. lonquimayensis $+L$. shitan, but these findings are preliminary, since there are no Cyt-b sequences in GenBank for some species currently assigned to the $L$. elongatus clade (L. carlosgarini, L. crandalli and $L$. scorialis). A future study with additional species could yield a different topology. Moreover, a limitation in our study is the use of a single mtDNA marker, one limitation also shared by almost all recent descriptions of Liolaemus (sensu stricto). For example, hybridization and introgression have been found in closely related species of Liolaemus (Olave et al. 2011) and a future study of the species described here using nuclear markers would be greatly desirable. Besides, the clade formed by L. elongatus + L. lonquimayensis $+L$. shitan requires a deeper analysis. A sample of $L$. shitan does not form a monophyletic haploclade with respect to $L$. elongates. A work published previously to the description of this species showed that this "dark phenotype" from San Antonio del Cuy (25 de Mayo, Argentina) is not genetically distinctive enough to consider it as candidate species (Morando et al. 2003: 178) and it has been suggested as possible synonym of $L$. elongatus by Avila et al. (2015). However, since there are currently no DNA data for $L$. shitan from the type locality (Estancia Piedras Blancas, 25 de Mayo), we tentatively accept this species as valid. In regard to L. lonquimayensis, we believe that the relationship between this taxon and $L$. elongatus is not solved. The distinction between them is based in two features: absence of precloacal pores in the males of $L$. lonquimayensis and the fact that the four type specimens of $L$. lonquimayensis form a clade separated from L. elongatus samples (EscobarHuerta et al. 2015). However, Escobar-Huerta et al. (2015) used three sequences of $L$. elongatus in their phylogeny (BYU 47101, MVZ 232399 and BYU 47092). We used thirteen sequences of $L$. elongatus and one sequence of $L$. shitan and obtained a different result, $L$. lonquimayensis does not form a distinctive clade from $L$. elongatus (Figs 1 and 2). In this work, we include in the PCA analysis specimens of $L$. cf. elongatus from Llaima Volcano, located between the type locality of $L$. lonquimayensis and the northern limit of L. elongatus (Morando et al. 2003). Based on coloration and morphology, these specimens can be assigned to $L$. elongatus, although there are no molecular data to confirm this.

The second new species that is described here, Liolaemus leftrarui, is notable for the absence of precloacal pores and its light blue dorsal dots, because precloacal pores in males a typical feature in Liolaemus (Esquerré et al. 2013). The absence of precloacal pores also 
occurs in L. villaricensis (Torres-Pérez et al. 2009), the most closely related species to L. leftrarui. However, molecular evidence indicates that the species of the subgenus Liolaemus with complete absence of precloacal pores in males are paraphyletic and not monophyletic, as has been previously proposed (Cei and Videla 2003). For example, in our phylogeny $L$. leftrarui, $L$. neuquensis, $L$. punmahuida and $L$. tregenzai do not constitute a monophyletic group and none of them is the sister species of the others (although precloacal pores are lacking in all these species). Pincheira-Donoso and Núñez (2005) recorded L. villaricensis from Lonquimay volcano, but no specimens were deposited in an institutional collection. Here we add a second record of this species from this locality (SSUC Re 729-31).

Certainly, there is still much to be discovered about the diversity of the species of Liolaemus in southern and central Chile, especially in the Liolaemus elongatus-kriegi complex and the species related to L. villaricensis, for which several taxonomic issues still remain unsolved.

\section{Acknowledgements}

We thank Damien Esquerré for his help with the PCA analysis and providing a draft for the R script to perform the analysis and for his invaluable comments to the text. We thank the editor and the two anonymous reviewers who helped us to improve the manuscript. We thank P. Zavala (Pontificia Universidad de Católica de Chile) for allowing us to review and deposit material in the collection under his care. We are grateful to the following colleagues and institutions for allowing us to review specimens: M. Lamborot (LCUC), J. Artigas (MZUC) and H. Núnez (MNHNCL). Gustavo Escobar-Huerta for send us DNA data of L. lonquimayensis. Cesar Aguilar and Jack Walter Sites Jr. send us DNA data of one of the two specimens of $L$. leftrarui included in the phylogeny; which was generated as part of a work to be published elsewhere (Aguilar et al. in prep.). We thank especially Richard Etheridge (San Diego State University) for all his comments and improving the English. F. Lobo, L.J. Avila, F. Tillack, C. Garín, and A. Laspiur for sending literature. J. Troncoso-Palacios thanks M. Penna and A. Labra for their support. Thanks to F. Urra and E. Villarroel for their assistance in the field. Edvin Riveros-Riffo thanks M. Riveros and F. Ibieta for calling to his attention the presence of $L$. janequeoae during ascent to Laguna Verde. Thanks to the Servicio Agrícola y Ganadero (SAG) for the collecting permit ( $\mathrm{N}^{\circ} 4468$ ). Funding Cochilco-FONDECYT 1100995 (AE), Millennium Institute in Immunology and Immunotherapy, P09-016-F (AE), Nucleo UNAB DI-741-15/N (AE).

\section{References}

Abdala CS, Quinteros AS, Scrocchi GJ, Stazzonelli JC (2010) Three new species of the Liolaemus elongatus group (Iguania: Liolaemidae) from Argentina. Cuadernos de Herpetología 24(2): 93-109. 
Abdala CS, Quinteros AS (2014) Los últimos 30 años de estudios de la familia de lagartijas más diversa de Argentina. Actualización taxonómica y sistemática de Liolaemidae. Cuadernos de Herpetología 28(2): 55-82.

Avila LJ, Pérez CHF, Morando M (2003) A new species of Liolaemus (Squamata: Iguania: Liolaemidae) from northwestern Patagonia (Neuquén, Argentina). Herpetologica 59: 532-543. doi: $10.1655 / 02-67$

Avila LJ, Morando M, Pérez DR, Sites JW Jr (2004) Phylogenetic relationships of lizard of the Liolaemus petrophilus group (Squamata: Liolaemidae), with description of two new species from western Argentina. Herpetologica 60: 187-203. doi: 10.1655/03-04

Avila LJ, Morando M, Pérez DR, Sites JW Jr (2010) A new species of the Liolaemus elongatus clade (Reptilia: Iguania: Liolaemini) from Cordillera del Viento, northwestern Patagonia, Neuquén, Argentina. Zootaxa 2667: 28-42.

Avila LJ, Pérez CHF, Medina CD, Sites JW Jr, Morando M (2012) A new species of lizard of the Liolaemus elongatus clade (Reptilia: Iguania: Liolaemini) from Curi Leuvu River Valley, northern Patagonia, Neuquén, Argentina. Zootaxa 3325: 37-52.

Avila LJ, Martínez LE, Morando M (2013) Checklist of lizards and amphisbaenians of Argentina: an update. Zootaxa 3616(3): 201-238. doi: 10.11646/zootaxa.3616.3.1

Avila LJ, Medina CD, Pérez CHF, Sites JW Jr, Morando M (2015) Molecular phylogenetic relationships of the lizard clade Liolaemus elongatus (Iguania: Liolaemini) with the description of a new species from an isolated volcanic peak in northern Patagonia. Zootaxa 3947: 67-84. doi: 10.11646/zootaxa.3947.1.4

Breitman MF, Avila LJ, Sites JW Jr, Morando M (2012) How lizards survived blizzards: phylogeography of the Liolaemus lineomaculatus group (Liolaemidae) reveals multiple breaks and refugia in southern Patagonia, and their concordance with other co-distributed taxa. Molecular Ecology 25: 6068-6085. doi: 10.1111/mec.12075

Cei JM, Videla F (2003) A new species of Liolaemus lacking precloacal pores in males from the Andean south-eastern mountains of Mendoza Province, Argentina. (Liolaemidae, Iguania, Lacertilia, Reptilia). Bollettino del Museo Regionale di Scienze Naturali, Torino 20: 275-290.

Coura R (2005) An alternative protocol for DNA extraction from formalin fixed and paraffin wax embedded tissue. Journal of Clinical Pathology 58(8): 894-895. doi: 10.1136/ jcp.2004.021352

Darriba D, Taboada GL, Doallo R, Posada D (2012) jModelTest 2: More models, new heuristics and parallel computing. Nature Methods 9(8): 772. doi: 10.1038/nmeth.2109

Edgar RC (2004) MUSCLE: multiple sequence alignment with high accuracy and high throughput. Nucleic Acids Research 32(5): 1792-97. doi: 10.1093/nar/gkh340

Escobar-Huerta G, Santibáńez-Toro J, Ortiz JC (2015) Liolaemus lonquimayensis (Squamata: Liolaemidae), a new lizard species for Chile without precloacal pores. Gayana 79(1): 94-101. doi: $10.4067 / \mathrm{s} 0717-65382015000100009$

Esquerré D, Núñez H, Scolaro JA (2013) Liolaemus carlosgarini and Liolaemus riodamas (Squamata: Liolaemidae), two new species of lizards lacking precloacal pores, from Andean areas of central Chile. Zootaxa 3619(4): 428-452. doi: 10.11646/zootaxa.3619.4.2 
Esquerré D, Troncoso-Palacios J, Garín CF, Núñez H (2014) The missing leopard lizard: Liolaemus ubaghsi sp. nov., a new species of the leopardinus clade (Reptilia: Squamata: Liolaemidae) from the Andes of the O'Higgins Region in Chile. Zootaxa 3815(4): 507-525. doi: 10.11646/zootaxa.3815.4.3

Etheridge R (1995) Redescription of Ctenoblepharys adspersa Tschudi, 1845, and the Taxonomy of Liolaeminae (Reptilia: Squamata: Tropiduridae). American Museum Novitates 3142: 1-34.

Felsenstein J, Kishino H (1993) Is there something wrong with the bootstrap on phylogenies? A reply to Hillis and Bull. Systematic Biology 42: 193-200. doi: 10.1093/sysbio/42.2.193 Gajardo R (1994) La vegetación natural de Chile: clasificación y distribución geográfica. Editorial Universitaria, Santiago, $165 \mathrm{pp}$.

Gelman A, Rubin DB (1992) Inference from iterative simulation using multiple sequences. Statistical Science 7: 457-511. doi: 10.1214/ss/1177011136

Guindon S, Gascuel O (2003) A simple, fast and accurate method to estimate large phylogenies by maximum-likelihood. Systematic Biology 52: 696-704. doi: 10.1080/10635150390235520

Hellmich W (1934) Die eidechsen Chiles insbesonders die gattung Liolaemus. Nach den sammlungen Goetsch-Hellmich. Abhandlungen. Bayerischen Akademie der Wissenschaften. Mathematisch-Naturwissenschaftliche Klasse 24: 1-140.

Huelsenbeck JP, Ronquist F (2001) MrBayes: Bayesian inference of phylogeny. Bioinformatics 17: 754-755. doi: 10.1093/bioinformatics/17.8.754

Laurent RF (1985) Segunda contribución al conocimiento de la estructura taxonómica del género Liolaemus Wiegmann (Iguanidae). Cuadernos de Herpetología 1: 1-37.

Lobo F (2005) Las relaciones filogenéticas dentro del grupo chiliensis (Iguania: Liolaemidae: Liolaemus): sumando nuevos caracteres y taxones. Acta Zoológica Lilloana 49: 67-89.

Morando M, Avila LJ, Sites JW Jr (2003) Sampling strategies for delimiting species: genes, individuals, and populations in the Liolaemus elongatus-kriegi complex (Squamata: Liolaemidae) in Andean-Patagonian South America. Systematic Biology 52: 159-185. doi: $10.1080 / 10635150390192717$

Núnez H (2007) Liolaemus frassinettii, nueva especie de lagartija para los Altos de Cantillana, Región Metropolitana (Reptilia: Sauria). Boletín del Museo Nacional de Historia Natural, Chile 56: 81-87.

Olave M, Martínez LE, Avila LJ, Sites JW Jr, Morando M (2011) Evidence of hybridization in the Argentinean lizards Liolaemus gracilis and Liolaemus bibronii (Iguania: Liolaemini): An integrative approach based on genes and morphology. Molecular Phylogenetics and Evolution 61: 381-391. doi: 10.1016/j.ympev.2011.07.006

Pincheira-Donoso D, Núñez H (2005) Las especies chilenas del género Liolaemus Wiegmann, 1834 (Iguania: Tropiduridae: Liolaeminae). Taxonomía, sistemática y evolución. Publicación Ocasional del Museo Nacional de Historia Natural, Chile 59: 1-486.

Pincheira-Donoso D, Scolaro JA (2007) Iguanian species-richness in the Andes of boreal Patagonia: evidence for an additional new Liolaemus lizard from Argentina lacking precloacal glands (Iguania: Liolaeminae). Zootaxa 1452: 55-68.

Ronquist F, Huelsenbeck JP (2003) MRBAYES 3: Bayesian phylogenetic inference under mixed models. Bioinformatics 19: 1572-1574. doi: 10.1093/bioinformatics/btg180 
RStudio Team (2015) RStudio: Integrated development for R. RStudio, Inc., Boston, MA. http://www.rstudio.com/

Scolaro JA, Videla F, Puig S, Marcus A (2007) Diferencias morfológicas y status taxonómico de las especies simpátricas Liolaemus coeruleus y Liolaemus neuquensis (Reptilia: Iguania: Liolaemidae). Multequina 16: 53-63.

Schulte JA II, Macey JR, Espinoza RE, Larson A (2000) Phylogenetic relationships in the iguanid lizard genus Liolaemus: multiple origins of viviparous reproduction and evidence for recurring Andean vicariance and dispersal. Biological Journal of Linnean Society 69: 75-120. doi: 10.1006/bijl.1999.0346

Tamura K, Stecher G, Peterson D, Filipski A, Kumar S (2013) MEGA6: Molecular Evolutionary Genetics Analysis version 6.0. Molecular Biology and Evolution 30: 2725-2729. doi: $10.1093 / \mathrm{molbev} / \mathrm{mst} 197$

Torres-Pérez F, Méndez MA, Benavides E, Moreno RA, Lamborot M, Palma RE, Ortiz JC (2009) Systematics and evolutionary relationships of the mountain lizard Liolaemus monticola (Liolaemini): how morphological and molecular evidence contributes to reveal hidden species diversity. Biological Journal of the Linnean Society 96: 635-650. doi: 10.1111/j.1095-8312.2008.01140.x

Troncoso-Palacios J, Díaz HA, Esquerré D, Urra FA (2015) Two new species of the Liolaemus elongatus-kriegi complex (Iguania, Liolaemidae) from Andean highlands of southern Chile. ZooKeys 500: 83-109. doi: 10.3897/zookeys.500.8725

Troncoso-Palacios J, Elorza AA, Puas GI, Alfaro-Pardo E (2016) A new species of Liolaemus related to L. nigroviridis from the Andean highlands of Central Chile (Iguania, Liolaemidae). ZooKeys 555: 91-114. doi: 10.3897/zookeys.555.6011

Uetz P, Hošek J (2015) The Reptile Database. http://www.reptile-database.org [accessed December, 22, 2015]

Valdecantos S, Martínez V, Labra A (2014) Comparative morphology of Liolaemus lizards precloacal glands. Acta Herpetologica 9(2): 147-158. 


\section{Supplementary material I}

\section{Appendices}

Authors: Jaime Troncoso-Palacios, Hugo A. Diaz, German I. Puas, Edvin Riveros-Riffo, Alvaro A. Elorza

Data type: Microsoft Word (docx)

Explanation note:

Appendix I: Museum codes of the specimens examined.

Appendix II: GenBank accession numbers of the specimens used for phylogenetic analysis.

Appendix III: Eigenvalues, the percentage of the total variance and the cumulative percentage of variance in each of the 13 PCs found by the PCA.

Appendix IV: Correlation of each variable with each of the first three PCs.

Copyright notice: This dataset is made available under the Open Database License (http://opendatacommons.org/licenses/odbl/1.0/). The Open Database License $(\mathrm{ODbL})$ is a license agreement intended to allow users to freely share, modify, and use this Dataset while maintaining this same freedom for others, provided that the original source and author(s) are credited. 